\title{
Tail Risk Premia and Return Predictability
}

\author{
Tim Bollerslev, Viktor Todorov and Lai Xu
}

CREATES Research Paper 2014-49 


\title{
Tail Risk Premia and Return Predictability*
}

\author{
Tim Bollerslev† $\quad$ Viktor Todorov $\ddagger$ and Lai $\mathrm{Xu} \S$ \\ First Version: May 9, 2014 \\ This Version: September 29, 2014
}

\begin{abstract}
The variance risk premium, defined as the difference between actual and risk-neutralized expectations of the forward aggregate market variation, helps predict future market returns. Relying on new essentially model-free estimation procedure, we show that much of this predictability may be attributed to time variation in the shape of the tails and compensation demanded by investors for bearing jump tail risk. Our results are consistent with the idea that the temporal variation in the separate diffusive and jump risk components of the variance risk premium may be associated with notions of timevarying economic uncertainty and changes in risk aversion, or market fears, respectively.
\end{abstract}

Keywords: Variance risk premium; time-varying jump tails; market sentiment and fears; return predictability.

JEL classification: C13, C14, G10, G12.

${ }^{*}$ The research was supported by a grant from the NSF to the NBER, and CREATES funded by the Danish National Research Foundation DNRF78 (Bollerslev). We are grateful to an anonymous referee for her/his very useful comments. We would also like to thank Caio Almeida, Reinhard Ellwanger and seminar participants at NYU Stern, the 2013 SETA Meetings in Seoul, South Korea, the 2013 Workshop on Financial Econometrics in Natal, Brazil, and the 2014 SCOR/IDEI conference on Extreme Events and Uncertainty in Insurance and Finance in Paris, France for their helpful comments and suggestions.

$\dagger$ Department of Economics, Duke University, Durham, NC 27708, and NBER and CREATES; e-mail: boller@duke.edu.

${ }^{\ddagger}$ Department of Finance, Kellogg School of Management, Northwestern University, Evanston, IL 60208; e-mail:v-todorov@northwestern.edu.

$\S$ Department of Economics, Duke University, Durham, NC 27708; e-mail: lai.xu@duke.edu. 
"When the VIX is high, it's time to buy, when the VIX is low, it's time to go."

Wall Street adage

\section{Introduction}

The VIX is popularly referred to by market participants as the "investor fear gauge." Yet, on average only a small fraction of the VIX is arguably attributable to market fears. We show that rather than simply buying (selling) when the VIX is high (low), the genuine fear component of the index provides a much better guide for making "good" investment decisions.

Volatility clustering in asset returns is ubiquitous. This widely documented temporal variation in volatility (Schwert, 2011; Andersen, Bollerslev, Christoffersen, and Diebold, 2013) represents an additional source of risk over and above the variation in the actual asset prices themselves. ${ }^{1}$ For the market as whole, this risk is also rewarded by investors, as directly manifest in the form of a wedge between the actual and risk-neutralized expectations of the forward variation of the return on the aggregate market portfolio (Bakshi and Kapadia, 2003). Not only is the variance risk premium on average significantly different from zero, like the variance itself it also fluctuates non-trivially over time (Carr and Wu, 2009; Todorov, 2010). Mounting empirical evidence further suggest that unlike the variance, the variance risk premium is useful for predicting future aggregate market returns over and above the predictability afforded by more traditional predictor variables such as the dividend-price and other valuation ratios, with the predictability especially strong over relatively short quarterly to annual horizons (Bollerslev, Tauchen, and Zhou, 2009). ${ }^{2}$

The main goals of the present paper are twofold. First, explicitly recognizing the prevalence of different types of market risks, we seek to nonparametrically decompose their sum

\footnotetext{
${ }^{1}$ Following the classical ICAPM of Merton (1973), variance risk has traditionally been associated with changes in the investment opportunity set, which in turn induce a hedging component in the asset demands.

${ }^{2}$ Recent studies corroborating and extending the predictability results in Bollerslev, Tauchen, and Zhou (2009) include Drechsler and Yaron (2011), Han and Zhou (2011) Du and Kapadia (2012), Eraker and Wang (2012), Almeida, Vicente, and Guillen (2013), Bekaert and Hoerova (2013), Bali and Zhou (2013), Camponovo, Scaillet, and Trojani (2013), Kelly and Jiang (2013), Li and Zinna (2014), Vilkov and Xiao (2013) and Bollerslev, Marrone, Xu, and Zhou (2014), among others. The empirical results in Andreou and Ghysels (2013) and Bondarenko (2014) also suggest that the variance risk premium cannot be explained by other traditional risk factors.
} 
total as embodied in the variance risk premium into separate diffusive and jump risk components with their own distinct economic interpretations. Second, relying on this new decomposition of the variance risk premium, we seek to clarify where the inherent market return predictability is coming from and how it plays out over different return horizons and for different portfolios with different risk exposures.

Extending the long-run risk model of Bansal and Yaron (2004) to allow for time-varying volatility-of-volatility, Bollerslev, Tauchen, and Zhou (2009) and Drechsler and Yaron (2011) have previously associated the temporal variation in the variance risk premium with notions of time-varying economic uncertainty. On the other hand, extending the habit formation type preferences of Campbell and Cochrane (1999), Bekaert and Engstrom (2010) and Bekaert, Hoerova, and Lo Duca (2012) have argued that the variance risk premium may be interpreted as a proxy for aggregate risk-aversion. Meanwhile, as emphasized by Bollerslev and Todorov (2011b), the variance risk premium formally reflects the compensation for two very different types of risks: continuous and discontinuous price moves. The possibility of jumps, in particular, adds an additional unique source of market variance risk stemming from the locally non-predictable nature of jumps. This risk is still present even if the investment opportunity set does not changing over time (i.e., even in a static economy with independent and identically distributed returns), and it remains a force over diminishing investment horizon (i.e., even for short time-intervals where the investment opportunity set is approximately constant). As discussed more formally below, these distinctly different roles played by the two types of risks allows us to uniquely identify the part of the variance risk premium attributable to market sentiment, or fears, from the compensation for jump tail risk.

Our estimation of the separate components of the variance risk premium builds on and extends the new econometric procedures recently developed by Bollerslev and Todorov (2014). The basic idea involves identifying the shape of the risk-neutral jump tails from the rate at which the prices of short maturity options decay for successively deeper out-of-the-money contracts. Having identified the shape of the tails, their levels are easily determined by the actual prices of the options. In contrast to virtually all parametric jump-diffusion models hitherto estimated in the literature, which restrict the shape of the tail decay to be constant over time, we show that the shapes of the nonparametrically estimated jump tails vary signif- 
icantly over time, and that this variation contributes non-trivially to the temporal variation of the variance risk premium. The statistical theory underlying our new estimation procedure is formally based on an increasing cross-section of options. Importantly, this allows for a genuine predictive analysis avoiding the look-ahead bias which invariably plagues other more traditional parametric-based estimation procedures relying on long-span asymptotics for the tail estimation.

The two separately estimated components of the variance risk premium each exhibit their own unique dynamic features. Although both increase during times of financial crisis and distress (e.g., the 1997 Asian crisis, the 1998 Russian default, the 2007-08 global financial crisis, and the 2010 European sovereign debt crisis), the component due to jump risk typically remains elevated for longer periods of time. ${ }^{3}$ By contrast, the part of the variance risk premium attributable to "normal" risks rises significantly during other time periods that hardly register in the jump risk component (e.g., the end of the dotcom era in 2002-03). Counter to the implications from popular equilibrium-based asset pricing models, nonparametric regression analysis also suggests that neither of the two components of the variance risk premium can be explained as nonlinear functions of the aggregate market volatility. ${ }^{4}$ Hence, nonlinearity of the pricing kernel cannot be the sole explanation for the previously documented predictability inherent in the variance risk premium. ${ }^{5}$

The distinctly different dynamic dependencies in the two components of the variance risk premium also naturally suggests that the return predictability for the aggregate market portfolio afforded by the total variance risk premium may be enhanced by separately considering the two components in the return predictability regressions. Our empirical results confirm this conjecture. In particular, we find that most of the predictability for the aggregate market portfolio previously ascribed to the variance risk premium stems from the jump tail risk component, and that this component drives out most of the predictability stemming from the part of the variance risk premium associated with "normal" sized price

\footnotetext{
${ }^{3}$ The overall level of the market volatility also tends to mean revert more quickly than the jump risk premia following all of these events.

${ }^{4}$ The habit persistence model of Campbell and Cochrane (1999), for example, and its extension in Du (2010), imply such a nonlinear relationship.

${ }^{5}$ Similarly, nonlinearity cannot explain the empirically weak mean-variance tradeoff widely documented in the literature; see, e.g., Bollerslev, Sizova, and Tauchen (2012) and the many references therein.
} 
fluctuations. Replicating the predictability regressions for the aggregate market portfolio for size, value, and momentum portfolios comprised of stocks sorted on the basis of their market capitalizations, book-to-market values, and past annual returns, we document even greater increases in the degree of return predictability by separately considering the two variance risk premium components. The predictability patterns for the corresponding zero-cost high-minus-low arbitrage portfolios are generally supportive of our interpretation of the two components of the variance risk premium as measures of time-varying economic uncertainty, or changes in the investment opportunity set, and changes in risk aversion, or market fears, respectively.

Our empirical findings specifically pertaining to the predictability of the aggregate market portfolio are related to other recent empirical studies, which have argued that various optionsbased measures of jump risk are useful for forecasting future market returns. Santa-Clara and Yan (2010), in particular, find that an estimate of the equity risk premium due to jumps, as implied from options and a one-factor stochastic volatility jump diffusion model, significantly predict subsequent market returns. Similarly, Andersen, Fusari, and Todorov (2013) relying on a richer multi-factor specification find that a factor directly related to the risk-neutral jump intensity helps forecast future market returns. Allowing for both volatility jumps and self-exciting jump intensities, Li and Zinna (2014) report that the predictive performance of the variance risk premium estimated within their model may be improved by separately considering the estimated jump component. All of these studies, however, rely on specific model structures and long time-span asymptotics for parameter estimation and extraction of the state variables that drive the jump and stochastic volatility processes. By contrast, our empirical investigations are distinctly non-parametric in nature, thus imbuing our findings with a built-in robustness against model misspecification. ${ }^{6}$ Moreover, our approach for

\footnotetext{
${ }^{6}$ A plethora of competing parametric models have been used in the empirical option pricing literature. For instance, while one factor models, as in, e.g., Pan (2002), Broadie, Chernov, and Johannes (2007), and SantaClara and Yan (2010), are quite common, the empirical evidence in Bates (2000), Christoffersen, Heston, and Jacobs (2009) among others, clearly suggests that multiple volatility factors are needed. Correspondingly, in models that do allow for jumps, the jump arrival rates are typically taken to be constant, although the estimates in Christoffersen, Jacobs, and Ornthanalai (2012), Andersen, Fusari, and Todorov (2013) among others, clearly point to time-varying jump intensities. Related to this, Duffie, Pan, and Singleton (2000), Eraker (2004) among others, further advocate allowing for volatility jumps. Moreover, despite ample empirical evidence favoring log-volatility formulations when directly modeling returns, virtually all parametric option pricing models have been based on either affine or linear-quadratic specifications.
} 
estimating the temporal variation in the jump tail risk measures is based on the crosssection of options at a given point-in-time, thus circumventing the usual concerns about structural-stability and "look-ahead" biases that invariably plague conventional parametricbased procedures.

Other related nonparametric-based approaches includes Vilkov and Xiao (2013), who argue that a conditional Value-at-Risk (VaR) type measure extracted from options with the help of Extreme Value Theory (EVT) predicts future market returns, although the predictability documented in that study is confined to relatively short weekly horizons. Also, Du and Kapadia (2012) find that a tail index measure for jumps defined as the difference between the sum of squared log-returns and the square of summed log-returns affords some additional predictability for the market portfolio over and above that of the variance risk premium. In contrast to these studies, the new nonparametric jump risk measures proposed and analyzed here are all economically motivated, with direct analogs in popular equilibrium consumption-based asset pricing models. Moreover, the predictability results for the market portfolio and the interpretation thereof are further corroborated by our new empirical findings pertaining to other portfolio sorts and priced risk factors.

The rest of the paper is organized as follows. Section 2 presents our formal setup and definitions of the variance risk premium and its separate components. We also discuss how the jump tail risk component manifests within two popular stylized equilibrium setups. Section 3 outlines our new estimation strategy for nonparametrically extracting the jump tails. Section 4 describes the data that we use in our empirical analysis. The actual estimation results for the new jump tail risk measured discussed in Section 5. Section 6 presents the results from the return predictability regressions, beginning with the aggregate market portfolio followed by the results for the different portfolio sorts and systematic risk factors. Section 7 concludes.

\section{General Setup and Assumptions}

The continuous-time dynamic framework, and corresponding variation measures, underlying our empirical investigations is very general. It encompasses almost all parametric asset pricing models hitherto used in the literature as special cases. 


\subsection{Returns and Variance Risk Premium}

Let $X_{t}$ denote the price of some risky asset defined on the filtered probability space $(\Omega, \mathcal{F}, \mathbb{P})$, where $\left(\mathcal{F}_{t}\right)_{t \geq 0}$ refers to the filtration. We will assume the following dynamic continuous-time representation for the instantaneous arithmetic return on $X$,

$$
\frac{d X_{t}}{X_{t-}}=a_{t} d t+\sigma_{t} d W_{t}+\int_{\mathbb{R}}\left(e^{x}-1\right) \widetilde{\mu}^{\mathbb{P}}(d t, d x),
$$

where the drift and diffusive processes, $a_{t}$ and $\sigma_{t}$, respectively, are both assumed to have càdlàg paths, but otherwise left unspecified, $W_{t}$ is a standard Brownian motion, and $\mu(d t, d x)$ is a counting measure for the jumps in $X$ with compensator $d t \otimes \nu_{t}^{\mathbb{P}}(d x)$, so that $\widetilde{\mu}^{\mathbb{P}}(d t, d x) \equiv$ $\mu(d t, d x)-d t \otimes \nu_{t}^{\mathbb{P}}(d x)$ is a martingale measure under $\mathbb{P}^{7}$

The continuously compounded return from time $t$ to $t+\tau$, say $r_{[t, t+\tau]} \equiv \log \left(X_{t+\tau}\right)-$ $\log \left(X_{t}\right)$, implied by the formulation in (2.1) may be expressed as,

$$
r_{[t, t+\tau]}=\int_{t}^{t+\tau}\left(a_{s}+q_{s}\right) d s+\int_{t}^{t+\tau} \sigma_{s} d W_{s}+\int_{t}^{t+\tau} \int_{\mathbb{R}} x \widetilde{\mu}^{\mathbb{P}}(d s, d x),
$$

where $q_{t}$ represents the standard convexity adjustment term associated with the transformation from arithmetic to logarithmic returns. Correspondingly, the variability of the price over the $[t, t+\tau]$ time-interval is naturally measured by the quadratic variation,

$$
Q V_{[t, t+\tau]}=\int_{t}^{t+\tau} \sigma_{s}^{2} d s+\int_{t}^{t+\tau} \int_{\mathbb{R}} x^{2} \mu(d s, d x) .
$$

Even though the diffusive price increments associate with $\sigma$ and the jumps controlled by the counting measure $\mu$ both contribute to the total variation of returns and the pricing thereof, they do so in distinctly different ways.

In order to more formally investigate the separate pricing of the diffusive and jump components, we will assume the existence of the alternative risk-neutral probability measure $\mathbb{Q}$, under which the dynamics of $X$ takes the form,

$$
\frac{d X_{t}}{X_{t-}}=\left(r_{f, t}-\delta_{t}\right) d t+\sigma_{t} d W_{t}^{\mathbb{Q}}+\int_{\mathbb{R}}\left(e^{x}-1\right) \widetilde{\mu}^{\mathbb{Q}}(d t, d x),
$$

where $r_{f, t}$ and $\delta_{t}$ refer to the instantaneous risk-free rate and the dividend yield, respectively, $W_{t}^{\mathbb{Q}}$ is a Brownian motion under $\mathbb{Q}$, and $\widetilde{\mu}^{\mathbb{Q}}(d t, d x) \equiv \mu(d t, d x)-d t \otimes \nu_{t}^{\mathbb{Q}}(d x)$ where $d t \otimes \nu_{t}^{\mathbb{Q}}(d x)$

\footnotetext{
${ }^{7}$ This implicitly assumes that $X_{t}$ does not have fixed times of discontinuities. This assumption is satisfied by virtually all asset pricing models hitherto used in the literature.
} 
denotes the compensator for the jumps under $\mathbb{Q}$. The existence of $\mathbb{Q}$ follows directly from the lack of arbitrage under mild technical conditions (see, e.g., the discussion in Duffie, 2001). Importantly, while the no-arbitrage condition restricts the diffusive volatility process $\sigma_{t}$ to be the same under the $\mathbb{P}$ and $\mathbb{Q}$ measures, the lack of arbitrage puts no restrictions on the $d t \otimes \nu_{t}^{\mathbb{Q}}(d x)$ jump compensator for the "medium" and "larger" (in absolute value) sized jumps. In that sense, the two different sources of risk manifest themselves in fundamentally different ways in the pricing of the asset.

Consider the (normalized by horizon) variance risk premium on $X$ defined by,

$$
V R P_{t, \tau}=\frac{1}{\tau}\left(\mathbb{E}_{t}^{\mathbb{P}}\left(Q V_{[t, t+\tau]}\right)-\mathbb{E}_{t}^{\mathbb{Q}}\left(Q V_{[t, t+\tau]}\right)\right) .
$$

This mirrors the definition of the variance risk premium most commonly used in the options pricing literature (see, e.g., Carr and Wu, 2009), where the difference is also sometimes referred to as a volatility spread (see, e.g., Bakshi and Madan, 2006). ${ }^{8}$ Let

$$
C V_{[t, t+\tau]}=\int_{t}^{t+\tau} \sigma_{s}^{2} d s
$$

denote the total continuous variation over the $[t, t+\tau]$ time-interval, and denote the corresponding total predictable jump variation under the $\mathbb{P}$ and $\mathbb{Q}$ probability measures by, ${ }^{9}$

$$
J V_{[t, t+\tau]}^{\mathbb{P}}=\int_{t}^{t+\tau} \int_{\mathbb{R}} x^{2} \nu_{s}^{\mathbb{P}}(d x) d s \quad J V_{[t, t+\tau]}^{\mathbb{Q}}=\int_{t}^{t+\tau} \int_{\mathbb{R}} x^{2} \nu_{s}^{\mathbb{Q}}(d x) d s .
$$

The variance risk premium may then be decomposed as,

$$
\begin{aligned}
V R P_{t, \tau} & =\frac{1}{\tau}\left(\mathbb{E}_{t}^{\mathbb{P}}\left(C V_{[t, t+\tau]}+J V_{[t, t+\tau]}^{\mathbb{P}}\right)-\mathbb{E}_{t}^{\mathbb{Q}}\left(C V_{[t, t+\tau]}+J V_{[t, t+\tau]}^{\mathbb{Q}}\right]\right) \\
& =\frac{1}{\tau}\left[\left(\mathbb{E}_{t}^{\mathbb{P}}\left(C V_{[t, t+\tau]}\right)-\mathbb{E}_{t}^{\mathbb{Q}}\left(C V_{[t, t+\tau]}\right)\right)+\left(\mathbb{E}_{t}^{\mathbb{P}}\left(J V_{[t, t+\tau]}^{\mathbb{P}}\right)-\mathbb{E}_{t}^{\mathbb{Q}}\left(J V_{[t, t+\tau]}^{\mathbb{P}}\right)\right)\right] \\
& +\frac{1}{\tau}\left(\mathbb{E}_{t}^{\mathbb{Q}}\left(J V_{[t, t+\tau]}^{\mathbb{P}}\right)-\mathbb{E}_{t}^{\mathbb{Q}}\left(J V_{[t, t+\tau]}^{\mathbb{Q}}\right)\right) .
\end{aligned}
$$

${ }^{8}$ This difference also corresponds directly to the expected payoff on a (long) variance swap contact. Empirically, the variance risk premium for the aggregate market portfolio as defined in (2.5) is on average negative. In the discussion of the empirical results below we will refer to our estimate of $-V R P_{t, \tau}$ as the variance risk premium for short.

${ }^{9}$ The quadratic variation due to jumps equals $\int_{t}^{t+\tau} \int_{\mathbb{R}} x^{2} \mu(d s, d x)$, which does not depend on the probability measure. $J V_{[t, t+\tau]}^{\mathbb{P}}$ and $J V_{[t, t+\tau]}^{\mathbb{Q}}$ denote the predictable components of the jump variation, which do depend on the respective probability measure. By contrast, for the continuous component $C V_{[t, t+\tau]}$ the quadratic variation and its predictable component coincide. 
The first parenthesis inside the square brackets on the right-hand-side involves the differences between the $\mathbb{P}$ and $\mathbb{Q}$ expectations of the continuous variation. Analogously, the second parenthesis inside the square brackets involves the differences between the $\mathbb{P}$ and $\mathbb{Q}$ expectations of the same $\mathbb{P}$ jump variation measure. These two terms account for the pricing of the temporal variation in the diffusive risk $\sigma_{t}^{2}$ and the jump intensity process $\nu_{t}^{\mathbb{P}}(d x)$, respectively. For the aggregate market portfolio, these differences in expectations under the $\mathbb{P}$ and $\mathbb{Q}$ measures are naturally associated with investors willingness to hedge against changes in the investment opportunity set. By contrast, the very last term on the right-hand-side in the above decomposition involves the difference between the expectations of the objective $\mathbb{P}$ and risk-neutral $\mathbb{Q}$ jump variation measures evaluated under the same probability measure $\mathbb{Q}$. As such, this term is effectively purged from the compensation for time-varying jump intensity risk. It has no direct analogue for the diffusive price component, but instead reflects the "special" treatment of jump risk. ${ }^{10}$

Without additional parametric assumptions about the underlying model structure it is generally impossible to empirically identify and estimate the separate diffusive and jump risk components. ${ }^{11}$ However, by focussing on the jump "tails" of the distribution, it is possible (under very weak additional semi-nonparametric assumptions) to estimate a measure that parallels the second term in the above decomposition and the part of the variance risk premium due to the special compensation for jump tail risk. Moreover, as we argue in the

${ }^{10}$ Formally, the total quadratic variation in (2.3) may alternatively be expressed as,

$$
Q V_{[t, t+\tau]}=\langle\log (X), \log (X)\rangle_{[t, t+\tau]}+\int_{t}^{t+\tau} \int_{\mathbb{R}} x^{2} \widetilde{\mu}^{\mathbb{P}}(d s, d x)
$$

where first term on the right-hand side corresponds to the so-called predictable quadratic variation, and the second term is a martingale; see e.g., Protter (2004). The first predictable quadratic variation term captures the risk associated with the temporal variation in the stochastic volatility and its analogue for the jumps; i.e., the jump intensity $\nu_{t}^{\mathbb{P}}(d x)$. The second martingale term associated with the compensated, or demeaned, jump process $\widetilde{\mu}^{\mathbb{P}}(d t, d x) \equiv \mu(d t, d x)-d t \otimes \nu_{t}^{\mathbb{P}}(d x)$ stems solely from the the fact that jumps, or price discontinuities, may occur. This term has no analogue for the diffusive price component. The "special" compensation for jumps refer to the price attached to this second term. In theory, all jumps, "small" and "large," will contribute to this term. Empirically, however, with discretely sample prices and options data, it is impossible to uniquely identify and distinguish the "small" jumps from continuous price moves. Hence, in our empirical investigations, we restrict our attention to the "special" compensation for jump tail risk.

${ }^{11}$ Andersen et al. (2013) have recently estimated the separate components based on a standard two-factor stochastic volatility model augmented with a third latent time-varying jump intensity factor. 
next section, this new measure may be interpreted as a proxy for investor fears. ${ }^{12}$

\section{$2.2 \quad$ Jump Tail Risk}

The general dynamic representations in (2.1) and (2.4) do not formally distinguish between different sized jumps. However, there is ample anecdotal as well as more rigorous empirical evidence that "large" sized jumps, or tail events, are viewed very differently by investors than more "normal" sized price fluctuations (see, e.g. Bansal and Shaliastovich, 2011, and the references therein). Motivated by this observation, we will focus on the pricing of unusually "large" sized jumps, with the notion of "large" defined in a relative sense compared to the current level of risk in the economy. ${ }^{13}$ Empirically, of course, without an explicit parametric model it would also be impossible to separately identify the "small" jump moves from the diffusive price increments.

Specifically, define the left and right risk-neutral jump tail variation over the $[t, t+\tau]$ time-interval by,

$$
L J V_{[t, t+\tau]}^{\mathbb{Q}}=\int_{t}^{t+\tau} \int_{x<-k_{t}} x^{2} \nu_{s}^{\mathbb{Q}}(d x) d s, \quad R J V_{[t, t+\tau]}^{\mathbb{Q}}=\int_{t}^{t+\tau} \int_{x>k_{t}} x^{2} \nu_{s}^{\mathbb{Q}}(d x) d s,
$$

where $k_{t}>0$ is a time-varying cutoff pertaining to the log-jump size. ${ }^{14}$ Let the corresponding left and right jump tail variation measures under the actual probability measure $\mathbb{P}$, say $L J V_{[t, t+\tau]}^{\mathbb{P}}$ and $R J V_{[t, t+\tau]}^{\mathbb{P}}$, be defined analogously from the $d t \otimes \nu_{t}^{\mathbb{P}}(d x)$ jump tail compensator. In parallel to the definition of the variance risk premium in (2.5), the (normalized by horizon) left and right jump tail risk premia are then naturally defined by,

$$
\begin{aligned}
& L J P_{t, \tau}=\frac{1}{\tau}\left(\mathbb{E}_{t}^{\mathbb{P}}\left(L J V_{[t, t+\tau]}^{\mathbb{P}}\right)-\mathbb{E}_{t}^{\mathbb{Q}}\left(L J V_{[t, t+\tau]}^{\mathbb{Q}}\right)\right), \\
& R J P_{t, \tau}=\frac{1}{\tau}\left(\mathbb{E}_{t}^{\mathbb{P}}\left(R J V_{[t, t+\tau]}^{\mathbb{P}}\right)-\mathbb{E}_{t}^{\mathbb{Q}}\left(R J V_{[t, t+\tau]}^{\mathbb{Q}}\right)\right),
\end{aligned}
$$

\footnotetext{
${ }^{12}$ Intuitively, for $\tau \downarrow 0$,

$$
\lim _{\tau \downarrow 0} V R P_{t, \tau}=\int_{\mathbb{R}} x^{2}\left(\nu_{t}^{\mathbb{P}}(d x)-\nu_{t}^{\mathbb{Q}}(d x)\right),
$$

corresponding to the second term on the right-hand-side in the decomposition of $V R P_{t, \tau}$, and the lack of compensation for changes in the investment opportunity set over diminishing horizons.

${ }^{13}$ That is, our definition of what constitute "large" sized jumps and our jump tail risk measures are relative as opposed to absolute concepts.

${ }^{14}$ The use of a time-varying cutoff $k_{t}$ for identifying the "large" jumps directly mirrors the use of a timevarying threshold linked to the diffusive volatility $\sigma_{t}$ in the tests for jumps based on high-frequency intraday data pioneered by Mancini (2001).
} 
both of which contribute to $V R P_{t, \tau}$. Correspondingly, the difference $V R P_{t, \tau}-\left(L J P_{t, \tau}+\right.$ $\left.R J P_{t, \tau}\right)$ may be interpreted as the part of the variance risk premium attributable to "normal" sized price fluctuations.

Mimicking the decomposition of the variance risk premium discussed in the previous section, the left and right tail jump premia defined above may be decomposed as,

$$
L J P_{t, \tau}=\frac{1}{\tau}\left[\mathbb{E}_{t}^{\mathbb{P}}\left(L J V_{[t, t+\tau]}^{\mathbb{P}}\right)-\mathbb{E}_{t}^{\mathbb{Q}}\left(L J V_{[t, t+\tau]}^{\mathbb{P}}\right)\right]+\frac{1}{\tau}\left[\mathbb{E}_{t}^{\mathbb{Q}}\left(L J V_{[t, t+\tau]}^{\mathbb{P}}\right)-\mathbb{E}_{t}^{\mathbb{Q}}\left(L J V_{[t, t+\tau]}^{\mathbb{Q}}\right)\right],
$$

and

$$
R J P_{t, \tau}=\frac{1}{\tau}\left[\mathbb{E}_{t}^{\mathbb{P}}\left(R J V_{[t, t+\tau]}^{\mathbb{P}}\right)-\mathbb{E}_{t}^{\mathbb{Q}}\left(R J V_{[t, t+\tau]}^{\mathbb{P}}\right)\right]+\frac{1}{\tau}\left[\mathbb{E}_{t}^{\mathbb{Q}}\left(R J V_{[t, t+\tau]}^{\mathbb{P}}\right)-\mathbb{E}_{t}^{\mathbb{Q}}\left(R J V_{[t, t+\tau]}^{\mathbb{Q}}\right)\right],
$$

respectively. The first term on the right-hand-side in each of the two expressions involves the difference between the $\mathbb{P}$ and $\mathbb{Q}$ expectations of the same jump variation measures. Again, this directly mirrors the part of the variance risk premium associated with the difference between the $\mathbb{P}$ and $\mathbb{Q}$ expectations of the future diffusive risk $C V_{[t, t+\tau]}$. By contrast, the second term on the right-hand-side in each of the two expressions involves the difference between the expectations of the respective $\mathbb{P}$ and $\mathbb{Q}$ jump tail variation measures under the same probability measure $\mathbb{Q}$, reflecting the "special" treatment of jump tail risk. ${ }^{15}$

Under the additional assumption that the $\mathbb{P}$ jump intensity process is approximately symmetric for "large" sized jumps, the first term on the right-hand-side in the above decompositions of $L J P_{t, \tau}$ and $R J P_{t, \tau}$ will be approximately the same. ${ }^{16}$ Hence, for sufficiently large values of the cutoff $k_{t}$, the difference between the two jump tail premia,

$L J P_{t, \tau}-R J P_{t, \tau} \approx \frac{1}{\tau}\left[\mathbb{E}_{t}^{\mathbb{Q}}\left(L J V_{[t, t+\tau]}^{\mathbb{P}}\right)-\mathbb{E}_{t}^{\mathbb{Q}}\left(L J V_{[t, t+\tau]}^{\mathbb{Q}}\right)\right]-\frac{1}{\tau}\left[\mathbb{E}_{t}^{\mathbb{Q}}\left(R J V_{[t, t+\tau]}^{\mathbb{P}}\right)-\mathbb{E}_{t}^{\mathbb{Q}}\left(R J V_{[t, t+\tau]}^{\mathbb{Q}}\right)\right]$,

will be largely void of the compensation for temporal variation in jump intensity risk. As such, $L J P_{t, \tau}-R J P_{t, \tau}$ may be interpreted as a proxy for investor fears. This mirrors the

\footnotetext{
${ }^{15}$ In parallel to the expression for the variance risk premium above, it follows that for $\tau \downarrow 0$,

$$
\lim _{\tau \downarrow 0} L J P_{t, \tau}=\int_{x<-k_{t}} x^{2}\left(\nu_{t}^{\mathbb{P}}(d x)-\nu_{t}^{\mathbb{Q}}(d x)\right), \quad \lim _{\tau \downarrow 0} R J P_{t, \tau}=\int_{x>k_{t}} x^{2}\left(\nu_{t}^{\mathbb{P}}(d x)-\nu_{t}^{\mathbb{Q}}(d x)\right),
$$

corresponding to the second term on the right-hand-side in the respective decompositions.

${ }^{16}$ The assumption that the $\mathbb{P}$ jump intensity process is approximately symmetric deep in the tails is supported empirically by the EVT-based estimates for the S\&P 500 market portfolio reported in Bollerslev and Todorov (2011a).
} 
arguments behind the investor fear index proposed by Bollerslev and Todorov (2011b). ${ }^{17}$ However, in contrast to the estimates reported in Bollerslev and Todorov (2011b), which restrict the shape of the jump tails to be time-invariant, we explicitly allow for empirically more realistic time-varying tail shape parameters, relying on the information in the crosssection of options for identifying the temporal variation in the $\mathbb{Q}$ jump tails.

Going one step further, it follows readily that for approximately symmetric $\mathbb{P}$ jump tails,

$$
L J P_{t, \tau}-R J P_{t, \tau} \approx \frac{1}{\tau} \mathbb{E}_{t}^{\mathbb{Q}}\left(R J V_{[t, t+\tau]}^{\mathbb{Q}}\right)-\frac{1}{\tau} \mathbb{E}_{t}^{\mathbb{Q}}\left(L J V_{[t, t+\tau]}^{\mathbb{Q}}\right),
$$

thus expressing the fear component of the tail risk premia as a function of the $\mathbb{Q}$ jump tails alone. $^{18}$ As such, this conveniently avoids any tail estimation under $\mathbb{P}$, which inevitably is plagued by a dearth of "large" sized jumps and a "law-of-small-numbers," or Peso-type problem. Moreover, for the aggregate market portfolio the magnitude of the risk-neutral left jump tail dwarfs that of the right jump tail. Hence, empirically $L J P_{t, \tau}-R J P_{t, \tau}$ is approximately equal to the $\mathbb{Q}$ expectation of the negative left jump variation only,

$$
L J P_{t, \tau}-R J P_{t, \tau} \approx-\frac{1}{\tau} \mathbb{E}_{t}^{\mathbb{Q}}\left(L J V_{[t, t+\tau]}^{\mathbb{Q}}\right)
$$

affording a particularly simple expression for the fear component.

\subsection{Equilibrium Interpretations of the Jump Tail Measures}

The definition of the jump tail risk premia and their interpretation discussed above hinge solely on the general continuous-time specification for the price process in (2.1) and the corresponding no-arbitrage condition. Importantly, our empirical estimation of the different measures also do not require us to to specify any other aspects of the underlying economy. Nonetheless, in order to gain some intuition for the different measures, and $L J P_{t, \tau}$ in partic-

\footnotetext{
${ }^{17}$ A similar decomposition has recently been explored by Li and Zinna (2014) within a more restrictive fully parametric framework. The interpretation of the difference between the left and right jump tail variation as a proxy for investor fears is also broadly consistent with the stylized partial equilibrium model in Gabaix (2012), discussed further below, although the underlying one-factor representation does not formally distinguish between the different variation type measures explicitly defined here. Also, Schneider (2012) has shown that empirically the fear index is highly correlated with the fixed leg of a simple skew swap trading strategy.

${ }^{18}$ Of course, this same approximate expression for $L J P_{t, \tau}-R J P_{t, \tau}$ also holds true under assumption that the $\mathbb{Q}$ jump tails are orders of magnitude larger than the $\mathbb{P}$ jump tails, even if the $\mathbb{P}$ jump tails are not necessarily symmetric. For the values of the cutoff $k_{t}$ used in the empirical analysis below this is clearly the case.
} 
ular, we will briefly consider their manifestation within the context of two popular stylized equilibrium consumption-based asset pricing frameworks.

To begin, we consider a setup build on a representative agent with time non-separable Epstein-Zin preferences and affine dynamics for consumption and dividends. This setup has been analyzed extensively by Eraker and Shaliastovich (2008). It includes the longrun risks models of Bansal and Yaron (2004) and Drechsler and Yaron (2011), as well as the rare disaster model with time-varying probabilities for disasters of Gabaix (2012) and Wachter (2013) as special cases. In this general setup, the jump intensity under the statistical probability measure $\mathbb{P}$ may be conveniently expressed as,

$$
\nu_{t}^{\mathbb{P}}(d x)=\left(\nu_{t, 1}^{\mathbb{P}} * \cdots * \nu_{t, i}^{\mathbb{P}} * \cdots * \nu_{t, n}^{\mathbb{P}}\right)(d x)
$$

where $*$ denotes the convolution operator, $\nu_{t, i}^{\mathbb{P}}$ controls the intensity of different sources of jumps in the economy (e.g., jumps in consumption growth), which by assumption takes the form,

$$
\nu_{t, i}^{\mathbb{P}}(x)=\left(\alpha_{i}^{\prime} \mathbf{V}_{t}\right) \nu_{i}^{\mathbb{P}}(x)
$$

for some time-invariant jump intensity measures $\nu_{i}^{\mathbb{P}}(x)$ and the $\mathbf{V}_{t}$ vector of state variables that drive the dynamics of the fundamentals in the economy. The pricing kernel in this economy in turn implies that the jump intensity process under the risk-neutral probability measure $\mathbb{Q}$ takes the form,

$$
\nu_{t}^{\mathbb{Q}}(d x)=\left(\nu_{t, 1}^{\mathbb{Q}} * \cdots * \nu_{t, i}^{\mathbb{Q}} * \cdots * \nu_{t, n}^{\mathbb{Q}}\right)(d x)
$$

where

$$
\nu_{t, i}^{\mathbb{Q}}(x)=e^{\lambda_{i} x} \nu_{t, i}^{\mathbb{P}}(x)
$$

Comparing (2.9) and (2.10) with (2.11) and (2.12), the pricing of all jump risk in this economy is formally based on exponential tilting of the $\mathbb{P}$ jump distribution, with the extent of the tilting and the pricing of the different sources of risks determined by the $\lambda_{i}$-s. The actual values of the $\lambda_{i}$-s will depend on the structural parameters and the risk aversion of the representative agent in particular. Importantly, the temporal variation in the priced jump risk is driven by the same factors that drive the actual market jump risks. 
Further specializing this setup along the lines of the recent rare disaster models of Gabaix (2012) and Wachter (2013) involving a single source of (negative) jumps, the expression for the $\mathbb{Q}$ jump intensity simplifies to $\nu_{t}^{\mathbb{Q}}(x)=e^{-\gamma x} \nu_{t}^{\mathbb{P}}(x)$, where $\gamma$ refers to the risk-aversion of the representative agent. It follows readily from the definition of $L J P_{t, \tau}$ that in this situation,

$$
L J P_{t, \tau}=\frac{1}{\tau} \int_{t}^{t+\tau} \int_{\mathbb{R}} x^{2}\left(\mathbb{E}_{t}^{\mathbb{P}}\left(\nu_{s}^{\mathbb{P}}(d x)\right)-\mathbb{E}_{t}^{\mathbb{Q}}\left(\nu_{s}^{\mathbb{P}}(d x)\right)\right) d s+\frac{1}{\tau} \int_{t}^{t+\tau} \int_{\mathbb{R}}\left(1-e^{-\gamma x}\right) x^{2} \mathbb{E}_{t}^{\mathbb{Q}}\left(\nu_{s}^{\mathbb{P}}(d x)\right) .
$$

The second term on the right-hand-side arises solely from the representative agent's special attitude towards jump risk. Moreover, as this expression shows any variation in this term is intimately related to the state variables that drive the fundamentals in the economy.

As an alternative equilibrium framework, consider now the generalization of the habit formation model of Campbell and Cochrane (1999) recently proposed by Du (2010), in which the representative agent faces disaster risks in consumption. In this setup consumption growth is assumed to be i.i.d. and subject to the possibility of rare disasters in the form of extreme negative jumps, while the agent's risk-aversion $\gamma_{t}$ varies with the level of (external) habits determined by aggregate consumption. Correspondingly, the risk-neutral jump intensity may be expressed as,

$$
\nu_{t}^{\mathbb{Q}}(x)=f\left(\gamma_{t}\right) \nu_{t}^{\mathbb{P}}(d x)
$$

for some nonlinear function $f(\cdot)$. Within this model the pricing of jump risk is therefore directly related to $\gamma_{t}$ and the pricing of risk in the economy more generally. In contrast to the framework based on an agent with Epstein-Zin preferences, the jump distribution also does not change between the $\mathbb{P}$ and $\mathbb{Q}$ measures. Again, from the definition of $L J P_{t, \tau}$ it follows that in this situation,

$L J P_{t, \tau}=\frac{1}{\tau} \int_{t}^{t+\tau} \int_{\mathbb{R}} x^{2}\left(\mathbb{E}_{t}^{\mathbb{P}}\left(\nu_{s}^{\mathbb{P}}(d x)\right)-\mathbb{E}_{t}^{\mathbb{Q}}\left(\nu_{s}^{\mathbb{P}}(d x)\right)\right) d s+\frac{1}{\tau} \int_{t}^{t+\tau} \int_{\mathbb{R}} x^{2} \mathbb{E}_{t}^{\mathbb{Q}}\left[\left(1-f\left(\gamma_{s}\right)\right) \nu_{s}^{\mathbb{P}}(d x)\right] d s$.

Thus, unlike the Epstein-Zin setup discussed above where the temporal variation in the second term that reflects the special attitude towards jump risk is driven solely by $\nu_{t}^{\mathbb{P}}(x)$, this term now also varies explicitly with the time-varying risk-aversion of the representative agent. However, since $\nu_{t}^{\mathbb{P}}(x)$ and $f\left(\gamma_{t}\right)$ both depend nonlinearly on the risk-aversion coefficient, $L J P_{t, \tau}$ may simply be expressed as a nonlinear function of $\gamma_{t}$. The market volatility in this 
economy also depends nonlinearly on $\gamma_{t}$. Consequently, $L J P_{t, \tau}$ and the market volatility are effectively "tied" together in a nonlinear relationship.

Even though the exact form and interpretation of the $L J P_{t, \tau}$ measure differ across the different equilibrium settings, it clearly conveys a lot of information about the pricing of tail risk in the economy. The connection between the variation of $L J P_{t, \tau}$ with other observable quantities in the economy, such as the market volatility and jump intensity, can shed light on the validity of the different equilibrium models without the need to make any strong parametric assumptions. We turn next to a discussion of the new tail approximations and related estimation procedures that we use for empirically quantifying $L J P_{t, \tau}$ and the other tail risk measures introduced above.

\section{Jump Tail Estimation}

Our estimation of the $\mathbb{Q}$ jump tail measures builds on the specification for the $\nu_{t}^{\mathbb{Q}}(d x)$ jump intensity process proposed by Bollerslev and Todorov (2014),

$$
\nu_{t}^{\mathbb{Q}}(d x)=\left(\phi_{t}^{+} \times e^{-\alpha_{t}^{+} x} 1_{\{x>0\}}+\phi_{t}^{-} \times e^{-\alpha_{t}^{-}|x|} 1_{\{x<0\}}\right) d x .
$$

This specification explicitly allows the left $\left(^{-}\right)$and right $\left(^{+}\right)$jump tails to differ. Although it formally imposes the same structure on all sized jumps, the results that follow only requires that $\nu_{t}^{\mathbb{Q}}(x)$ satisfies (3.1) for "large" jumps beyond some threshold, say $|x|>k_{t}$.

The specification in (3.1) is very general, allowing for two separate sources of independent variation in the jump tails, in the form of "level shifts" governed by $\phi_{t}^{ \pm}$, and shifts in the rate of decay, or the "shape," of the tails governed by $\alpha_{t}^{ \pm}$. By contrast, the assumption of constant tail shape parameters, or $\alpha_{t}^{+}=\alpha_{t}^{-}=\alpha$, employed in essentially all parametric models estimated in the literature to date imply that the relative importance of differently sized jumps is time invariant, so that the only way for the intensity of "large" sized jumps to change over time is for the intensity of all sized jumps to change proportionally. ${ }^{19}$ In most models hitherto employed in the literature that do allow for temporal variation in the jump intensity process $\nu_{t}^{\mathbb{Q}}(d x)$, it is also assumed that the dynamic dependencies in the left and

\footnotetext{
${ }^{19}$ This includes the affine jump diffusion models of Duffie, Pan, and Singleton (2000), the time-changed tempered stable models of Carr, Geman, Madan, and Yor (2003), along with the nonparametric estimation procedure employed in Bollerslev and Todorov (2011b).
} 
right tails may be described by the identical level-shift process, with the temporal variation in $\phi_{t}^{+}=\phi_{t}^{-}$driven by a simple affine function of the diffusive variance $\sigma_{t}^{2} .{ }^{20}$ By contrast, the temporal variation in $\phi_{t}^{ \pm}$is left completely unspecified in the present setup.

The jump intensity process in (3.1) readily allows for closed-form solutions for the integrals that define $L J V_{[t, t+\tau]}^{\mathbb{Q}}$ and $R J V_{[t, t+\tau]}^{\mathbb{Q}}$ in equation (2.6) in terms of the $\alpha_{t}^{ \pm}$and $\phi_{t}^{ \pm}$ tail parameters and the cutoff $k_{t}$ defining "large" jumps. In particular, assuming that the tail parameters remain constant over the horizon $\tau$, the left and right jump tail variation measures may be succinctly expressed as,

$$
\begin{aligned}
& L J V_{[t, t+\tau]}^{\mathbb{Q}}=\tau \phi_{t}^{-} e^{-\alpha_{t}^{-}\left|k_{t}\right|}\left(\alpha_{t}^{-} k_{t}\left(\alpha_{t}^{-} k_{t}-2\right)+2\right) /\left(\alpha_{t}^{-}\right)^{3}, \\
& R J V_{[t, t+\tau]}^{\mathbb{Q}}=\tau \phi_{t}^{+} e^{-\alpha_{t}^{+}\left|k_{t}\right|}\left(\alpha_{t}^{+} k_{t}\left(\alpha_{t}^{+} k_{t}+2\right)+2\right) /\left(\alpha_{t}^{+}\right)^{3} .
\end{aligned}
$$

Our estimation of $\alpha_{t}^{ \pm}$and $\phi_{t}^{ \pm}$, and in turn the $L J V_{[t, t+\tau]}^{\mathbb{Q}}$ and $R J V_{[t, t+\tau]}^{\mathbb{Q}}$ measures, will be based on out-of-the-money (OTM) puts and calls for the left and right tails, respectively. Intuitively, the $\alpha_{t}^{ \pm}$parameters may be uniquely identified from the rate at which the prices of the options decay in the tail, while for given tail shapes the $\phi_{t}^{ \pm}$parameters may be inferred from the actual option price levels.

Formally, let $O_{t, \tau}(k)$ denote the time $t$ price of an OTM option on $X$ with time to expiration $\tau$ and log-moneyness $k$. It follows then from Bollerslev and Todorov (2011b) that for two put options with the same maturity $\tau \downarrow 0$, but different strikes $k_{1} \downarrow-\infty<k_{2} \downarrow-\infty$, $\log \left(O_{t, \tau}\left(k_{2}\right) / O_{t, \tau}\left(k_{1}\right)\right) \approx\left(1+\alpha_{t}^{-}\right)\left(k_{2}-k_{1}\right)$. Similarly, for two call options with strikes $k_{1} \uparrow$ $\infty<k_{2} \uparrow \infty, \log \left(O_{t, \tau}\left(k_{2}\right) / O_{t, \tau}\left(k_{1}\right)\right) \approx\left(1-\alpha_{t}^{+}\right)\left(k_{2}-k_{1}\right)$. Utilizing these approximations, Bollerslev and Todorov (2014) show how the time-varying tail shape parameters $\alpha_{t}^{ \pm}$may be consistently estimated from an ever increasing number of deep OTM short-maturity options by ${ }^{21}$

$$
\widehat{\alpha}_{t}^{ \pm}=\operatorname{argmin}_{\alpha^{ \pm}} \frac{1}{N_{t}^{ \pm}} \sum_{i=1}^{N_{t}^{ \pm}}\left|\log \left(\frac{O_{t, \tau}\left(k_{t, i}\right)}{O_{t, \tau}\left(k_{t, i-1}\right)}\right)\left(k_{t, i}-k_{t, i-1}\right)^{-1}-\left(1 \pm\left(-\alpha^{ \pm}\right)\right)\right|,
$$

where $N_{t}^{ \pm}$denotes the total number of calls (puts) used in the estimation with moneyness $0<k_{t, 1}<\ldots<k_{t, N_{t}^{+}}\left(0<-k_{t, 1}<\ldots<-k_{t, N_{t}^{-}}\right)$. In the results reported on below, we

\footnotetext{
${ }^{20}$ This approach is exemplified by the jump-diffusion models estimated in Pan (2002) and Eraker (2004).

${ }^{21}$ The use of a robust M-estimator effectively downweighs the influence of any "outliers."
} 
implement this estimator on a weekly basis, thus implicitly assuming that the $\alpha_{t}^{ \pm}$parameters only changes from week to week.

The estimates for $\alpha_{t}^{ \pm}$in (3.3) put no restrictions on the $\phi_{t}^{ \pm}$parameters that shift the level of the jump intensity process through time. Meanwhile, let $r_{t, \tau}$ denote the risk-free interest rate over the $[t, t+\tau]$ time-interval, and $F_{t, \tau}$ the time $t$ futures price of $X_{t+\tau}$. It then follows from Bollerslev and Todorov (2014) that for $\tau \downarrow 0$ and $k<0, e^{r t, \tau} O_{t, \tau}(k) / F_{t-, \tau} \approx$ $\tau \phi_{t}^{-} e^{k\left(1+\alpha_{t}^{-}\right)} /\left(\alpha_{t}^{-}\left(\alpha_{t}^{-}+1\right)\right)$, while for $k>0, e^{r t, \tau} O_{t, \tau}(k) / F_{t-, \tau} \approx \tau \phi_{t}^{+} e^{k\left(1-\alpha_{t}^{+}\right)} /\left(\alpha_{t}^{+}\left(\alpha_{t}^{+}-1\right)\right)$. Utilizing these approximations, the "level shift" parameters may be estimated in a second step by,

$$
\begin{array}{r}
\widehat{\phi}_{t}^{ \pm}=\operatorname{argmin}_{\phi^{ \pm}} \frac{1}{N_{t}^{ \pm}} \sum_{i=1}^{N_{t}^{ \pm}} \mid \log \left(\frac{e^{r_{t, \tau}} O_{t, \tau}\left(k_{t, i}\right)}{\tau F_{t-, \tau}}\right)-\left(1 \mp \widehat{\alpha}_{t}^{ \pm}\right) k_{t, i} \\
+\log \left(\widehat{\alpha}_{t}^{ \pm} \mp 1\right)+\log \left(\widehat{\alpha}_{t}^{ \pm}\right)-\log \left(\phi^{ \pm}\right) \mid .
\end{array}
$$

Taken together these estimates completely characterize the $\mathbb{Q}$ jump intensity process in (3.1), and in turn all of the jump tail risk measures defined in Section 2.

\section{Data}

The data used in our empirical analysis comes from three different sources. The raw options data is obtained from OptionMetrics, and consists of closing bid and ask quotes for all S\&P 500 options traded on the Chicago Board of Options Exchange (CBOE), along with the corresponding zero coupon rates. The options span the period from January 1996 to August 2013 , for a total of 4,445 trading days. ${ }^{22}$

The estimates for the jump tail parameters in (3.3) and (3.4) formally rely on an increasing number of arbitrarily short-lived OTM options to eliminate the impact of the diffusive price component. In an effort to best mimic this condition, we restrict our analysis to options with no more than 45 days until expiration. To help alleviate the impact of market microstructure complications for the shortest lived options, we also rule out any options with less than eight days to maturity. In practice, of course, for a given fixed maturity,

\footnotetext{
${ }^{22}$ Following standard "cleaning" procedures to rule out arbitrage, starting from the closest at-the-money options we omit any out-of-the-money options for which the midquotes do not decrease with the strike price.
} 
these OTM option prices will still reflect some diffusive risk. To help mitigate this risk, for the estimation of the left jump tail parameters, we only use puts with log-moneyness less than minus two-and-a-half times the maturity-normalized Black-Scholes at-the-money implied volatility. Similarly, for the right jump tail parameters, we only use call options with log-moneyness in excess of the maturity-normalized Black-Scholes implied volatility. ${ }^{23}$ In the end, this leaves us with an average of 100.2 and 51.0 puts and calls per week, respectively, over the full sample.

Our construction of the actual realized variation measures and the variance risk premium rely on high-frequency S\&P 500 futures prices obtained from Tick Data Inc. The intraday prices are recorded at five-minute intervals, starting at 8:35 CST until the last price of the day at 15:15 CST, for a total of 81 observations per trading day. We also use these same highfrequency data in testing whether the option-based $\mathbb{Q}$ jump tail expectations are consistent with the subsequently observed $\mathbb{P}$ jump tail realizations.

Our aggregate market return predictability regressions are based on a broad valueweighted portfolio of all CRSP firms incorporated in the U.S. and listed on the NYSE, AMEX, or NASDAQ stock exchanges. The relevant time series of daily returns are obtained from Kenneth R. French's data library. ${ }^{24}$ We also rely on that same data source for daily returns on various size, book-to-market and momentum sorted portfolios. Lastly, we obtain data on the monthly dividend-price ratio for the aggregate market from CRSP.

\section{Empirical Tail Measures}

The left and right $\mathbb{Q}$ jump variation measures introduced above, including the approximate fear component in (2.8), may all be expressed as explicit functions of the jump tail parameters in (3.1). We begin our empirical analysis with a discussion of these parameters and the timevarying left and right "large" jump intensities implied by the estimates.

\footnotetext{
${ }^{23}$ By explicitly relating the threshold of the moneyness for the options used in the estimation to the overall level of the volatility, we screen out more relatively close to at-the-money options in periods of high volatility, thereby effectively minimizing the impact of the on average larger diffusive price component in the OTM option price when the volatility is high. Since the market for call options is less liquid than the market for puts, we rely on a more lenient cutoff for the right tail estimation.

${ }^{24}$ Website: http://mba.tuck.dartmouth.edu/pages/faculty/ken.french.
} 


\subsection{Tail Parameters}

Our estimates for the weekly left and right jump tail "shape" parameters are based on equation (3.3) and all of the qualifying options within each calendar week. The resulting sample mean of $\hat{\alpha}_{t}^{-}$equals 16.23 compared to 61.81 for $\hat{\alpha}_{t}^{+}$, indicative of the on average much slower tail decay inherent in the put versus call OTM option prices. Further to this effect, the top two panels in Figure 1 show $1 / \hat{\alpha}_{t}^{ \pm}$corresponding to the left and right jump tail indexes. The estimates for the left tail index varies almost ten-fold over the sample, ranging from a low of around 0.03 in 1997 and 2007, to a high of more than 0.25 in 2008-09 at the height of the recent financial crisis. Although less dramatic, the estimates for the right tail index also exhibit substantial variation over time. These temporal dependencies are directly manifest in the form of first order autocorrelations for the left and right tail "shape" parameters equal to 0.59 and 0.67 , respectively. ${ }^{25}$

The jump intensity process, of course, also depends on the "level" parameters. Our weekly estimates for these are based on the expression in (3.4). Rather than plotting the estimates for $\phi_{t}^{ \pm}$, the bottom two panels in Figure 1 show the annualized left and right "large" jump intensities implied by $\widehat{\alpha}_{t}^{ \pm}$and $\widehat{\phi}_{t}^{ \pm}$,

$$
L J I_{t}=\int_{x<-\left|k_{t}\right|} \nu_{t}^{\mathbb{Q}}(d x)=\widehat{\phi}_{t}^{-} e^{-\widehat{\alpha}_{t}^{-}\left|k_{t}\right|} / \widehat{\alpha}_{t}^{-}, \quad R J I_{t}=\int_{x>\left|k_{t}\right|} \nu_{t}^{\mathbb{Q}}(d x)=\widehat{\phi}_{t}^{+} e^{-\widehat{\alpha}_{t}^{+}\left|k_{t}\right|} / \widehat{\alpha}_{t}^{+}
$$

The calculation of these measures also necessitates a choice for the cutoff $k_{t}$ pertaining to the log-jump size and the start of the jump "tails." For both of the plots in the figure, as well as the $R J V_{t}$ and $L J V_{t}$ jump variation measures reported on below, we fix $k_{t}$ at 6.868 times the normalized Black-Scholes ATM volatility at time $t$. This specific cutoff corresponds to the median strike price for the deepest OTM puts in the sample. ${ }^{26}$

Allowing the $\alpha_{t}^{ \pm}$tail "shape" parameters to vary over time, results in fairly stable and

\footnotetext{
${ }^{25}$ Our finding of time-varying $\alpha_{t}^{ \pm}$parameters is consistent with the evidence for serially correlated "extreme" returns based on the so-called extremogram estimator in Davis and Mikosch (2009) and Davis et al. (2012). The recent cross-sectional based tail index estimates reported in Chollete and Lu (2011), Kelly and Jiang (2013) and Ruenzi and Weigert (2011) also point to strong dynamic dependencies. All of these studies, however, pertain to the actual return distributions and the shape of the tails under $\mathbb{P}$. Recent studies that have estimated somewhat simpler dynamic dependencies in the tails under $\mathbb{Q}$ include Almeida, Vicente, and Guillen (2013), Du and Kapadia (2012), Hamidieh (2011), Siriwardane (2013), and Vilkov and Xiao (2013).

${ }^{26}$ We also experimented with other choices for this "tail" cutoff, resulting in qualitatively very similar dynamic features and predictability regressions to the ones reported below. Further details concerning these additional results are available in a Supplementary Appendix.
} 
mildly serially correlated intensities for the "large" negative jumps. Meanwhile, there is a sense of "euphoria" and relatively high jump intensities for the "large" positive jumps embedded in the OTM call option prices leading up to the financial crisis. Of course, the right jump tail intensities are orders of magnitude less than those for the left jump tail. We turn next to a discussion of the jump tail variation measures and risk premia implied by these estimates for the $\nu_{t}^{\mathbb{Q}}(d x)$ "large" jump intensity process.

\subsection{Jump Tail Variation Measures}

Our estimates for the weekly left and right $\mathbb{Q}$ jump variation measures, as implied by equation (3.2), are depicted in Figure 2. Looking first at $L J V_{t}$ in the top panel, the measure inherits many of the same key dynamic dependencies evident in the left tail index shown in the top left panel in Figure 1. However, referring to Panel B in Table 1, the sample correlation between $L J V_{t}$ and $L J I_{t}$ is only equal to 0.26. By contrast, the correlation between $R J V_{t}$ and the right tail intensity $R J I_{t}$ equals 0.89 . Of course, as Figure 2 and Table 1 both make clear, $R J V_{t}$ is orders of magnitude less than $L J V_{t}$, so the fear component defined as the difference between the two is effectively equal to $-L J V_{t}$, as previously stated in (2.8).

To underscore the importance of explicitly allowing both the "shape" and the "level" of the jump tails to change over time in the estimation of this new fear component, the left panel in Figure 3 shows the estimates for the left jump tail variation $L J V_{t}^{*}$ obtained by restricting $\alpha_{t}^{-}=\alpha^{-}$to be constant, but allowing $\phi_{t}^{-}$to change over time. Correspondingly, the right panel shows the estimates for $L J V_{t}^{* *}$ obtained by restricting $\phi_{t}^{-}=\phi^{-}$to be constant, but allowing $\alpha_{t}^{-}$to be time-varying. Restricting the "shape" parameter to be constant, as is commonly done in the literature, clearly mutes the temporal variation and cuts the sample standard deviation of $L J V_{t}^{*}$ in half compared to $L J V_{t}$. By contrast, restricting the temporal variation to be solely driven by the "shape" of the jump tails, results in an even more dramatic increase in the magnitude of the fear component during the recent financial crisis. Along these lines, it is also worth noting that the first order sample autocorrelation for $L J V_{t}$ is larger than the autocorrelations of both $L J V_{t}^{*}$ and $L J V_{t}^{* *}$. Consistent with the return

predictability results discussed below, $L J V_{t}$ also correlates more strongly with $L J V_{t}^{* *}$ than 
$L J V_{t}^{*} \cdot 27$

The stylized equilibrium models discussed in Section 2.3 imply that the variation in $L J V_{t}$, is a direct, possibly nonlinear, function of the spot volatility. To investigate this conjecture empirically, Figure 4 presents the results from a nonparametric kernel regression of our nonparametric estimate of $L J V_{t}$ on the at-the-money implied variance from the shortestmaturity options available on the day, where the latter serves as a proxy for the unobservable spot volatility. ${ }^{28}$ As the figure shows, there is a substantial amount of variation in $L J V_{t}$ that cannot be explained by the current market volatility, even when allowing for a highly nonlinear relation between the two series. Further, as directly seen from the right panel in Figure 4, forcing $L J V_{t}$ to have the same value for a given market volatility produces a fitted variation measure with a much more pronounced spike than the actual $L J V_{t}$ series in the aftermath of the dot-com bubble and the mild economic recession in the early 2000s. On the other hand, since the spot volatility is generally faster mean reverting than the actual $L J V_{t}$ series, the nonlinear projection of $L J V_{t}$ on the volatility series results in a shorterlived impact of the recent financial crises. In sum, $L J V_{t}$ contains its own unique dynamic dependencies and isn’t simply spanned by the volatility.

\subsection{Jump Tail Variation and Return Correlations}

The sample correlations between the weekly returns on the aggregate market portfolio $M R K$ and the different jump tail variation measures, reported in the first row in Panel B of Table 1, are all negative. ${ }^{29}$ This mirrors the contemporaneous asymmetric return-volatility relationship, or so-called "leverage effect," widely documented in the literature for other volatility measures and models; see, e.g., the discussion in Bollerslev, Sizova, and Tauchen (2012) and the references therein. At the same time, the contemporaneous correlations between the

\footnotetext{
${ }^{27}$ The correlation between $L J V_{t}$ and the fear index estimated in Bollerslev and Todorov (2011b) relying on long-span asymptotics and the more restrictive assumption of constant tail "shape" parameters equals 0.75 .

${ }^{28}$ The reported kernel density estimates are based on a Gaussian kernel with the bandwidth parameter set according to the prescription in Bowman and Azzalini (1997). We also experimented with the use of alternative nonparametric estimates for the spot volatility obtained from high-frequency data on the S\&P 500 index futures, resulting in very similar nonparametric regression estimates for $L J V_{t}$.

${ }^{29} \mathrm{All}$ of the weekly variation measures are based on data available at the 15:15 CST close of the CBOE on Fridays, while the weekly aggregate market returns span the period from 16:00 EST the previous Monday to 16:00 EST the following Monday.
} 
different tail variation measures and the weekly returns on the $S M B, H M L$ and $W M L$ zero-cost portfolios, further analyzed below, are all smaller (in absolute value) and some even positive.

Meanwhile, with the exceptions of $R J V_{t}$, the sample correlations between the jump tail variation measures and the market return over the subsequent week, reported in the first row in Panel C of Table 1, are all positive. This suggests that a risk-return tradeoff, or "volatility feedback effect," may also be operative, whereby an increase (decrease) in one of the measures causes an immediate drop (rise) in the price in order to allow for higher (lower) future returns as a compensation for the increased (decreased) risk. Of course, these unconditional sample correlations do not distinguish whether the higher (lower) returns are indeed associated with an increase (decrease) in systematic risk or a change in the attitude towards risk, or both.

\subsection{Tail "Shape" Variation: Risk or Attitude to Risk?}

Our interpretation of $L J V_{t}$ as a measure of investor sentiment, or market fears, hinges on the standard no-arbitrage condition and the fact that it does not restrict the form of the $d t \otimes \nu_{t}^{\mathbb{Q}}(d x)$ jump compensator for the "large" sized jumps in (2.4) vis-a-vis the $d t \otimes \nu_{t}^{\mathbb{P}}(d x)$ jump compensator in (2.1). If, on the other hand, jumps and diffusive price moves were treated as identical risks by investors, the jump intensity process should be the same under the $\mathbb{P}$ and $\mathbb{Q}$ measures. Consequently, the mapping from $\nu_{t}^{\mathbb{P}}(d x)$ to $\nu_{t}^{\mathbb{Q}}(d x)$ directly reflects the "special" compensation for jump tail risk in the economy, as exemplified by the exponential tilting in equation (2.12) implied by the stylized long-run risk and rare disaster models discussed in Section 2.3, or the proportional shift from $\mathbb{P}$ to $\mathbb{Q}$ in equation (2.14) implied by the habit formation model.

The estimation of a general process for $\nu_{t}^{\mathbb{P}}(d x)$ that parallels that of $\nu_{t}^{\mathbb{Q}}(d x)$ in $(3.1)$ is inevitable plagued by a dearth of "large" jump tail realizations over short weekly time intervals. Instead, as a way to meaningfully test whether the "shape" of the risk-neutral and actual jump tails are indeed the same, as implied for example by the habit persistence model with measure change given in (2.14), we consider the time-series of actual high-frequencybased tail realizations over the full sample. In particular, let $\eta_{s}$ denote the threshold for 
defining the "large" negative jump realizations. Provided the jump tail "shape" parameter for $\nu_{t}^{\mathbb{P}}(d x)$ equals $\alpha_{t}^{-}$, the integral pertaining to the realized jumps,

$$
\int_{t}^{t+\tau} \int_{x<\eta_{s}}\left[x-\frac{e^{-\alpha_{t}^{-} \eta_{s}}\left(1+\alpha_{t}^{-} \eta_{s}\right)}{\left(\alpha_{t}^{-}\right)^{2}}\right] \mu(d s, d x)
$$

should then be a martingale under the statistical probability measure $\mathbb{P}^{30}$ Importantly, this condition does not depend on the overall "level" of the actual jump tails, but only on their "shape."

Substituting the weekly estimates of $\hat{\alpha}_{t}^{-}$for the $\mathbb{Q}$ jump tails in place of $\alpha_{t}^{-}$, the above martingale condition is readily operationalized as a test for identical jump tail "shapes" under the $\mathbb{P}$ and $\mathbb{Q}$ measures. Specifically, define the vector of sample moments,

$$
\widehat{\mathbf{m}}=\frac{1}{T}\left(\sum_{s \in[0, T): \Delta \log \left(X_{s}\right)<\eta_{s}}\left[\Delta \log \left(X_{s}\right)-\frac{e^{-\hat{\alpha}_{s}^{-} \eta_{s}}\left(1+\hat{\alpha}_{s}^{-} \eta_{s}\right)}{\left(\hat{\alpha}_{s}^{-}\right)^{2}}\right] \otimes \psi_{s}\right)
$$

where $\psi_{s}$ refers to any vector of valid instruments. Also, let $\widehat{W}$ denote an estimate for the corresponding asymptotic variance-covariance matrix for $\widehat{\mathbf{m}} \cdot{ }^{31}$ If the martingale condition is satisfied, $T \widehat{\mathbf{m}}^{\prime} \widehat{W}^{-1} \widehat{\mathbf{m}}$ should be (asymptotically for increasing sample size $T$ ) distributed as a Chi-square distribution with degrees of freedom equal to the dimension of the instrument vector $\psi_{s}$.

In implementing the test we rely on high-frequency intraday five-minute returns along with a time-varying threshold for determining the "large" sized jumps. ${ }^{32}$ In particular, fixing $\eta_{s}$ at six times the estimated continuous five-minute return variation, together with a twodimensional instrument vector comprised of a constant and an estimate of the integrated volatility over the previous day relative to the occurrence of a jump at time $s$, results in a test statistic of 170.6 , thus strongly rejecting the null hypothesis that diffusive and jump

\footnotetext{
${ }^{30} \mathrm{An}$ analogous martingale condition obviously holds for the right jump tails.

${ }^{31}$ By standard arguments, the covariance matrix may be consistently estimated by,$$
\widehat{W}=\frac{1}{T}\left(\sum_{s \in[0, T): \Delta \log \left(X_{s}\right)<\eta_{s}}\left(\Delta \log \left(X_{s}\right)-\frac{e^{-\hat{\alpha}_{s}^{-} \eta_{s}}\left(1+\hat{\alpha}_{s}^{-} \eta_{s}\right)}{\left(\hat{\alpha}_{s}^{-}\right)^{2}}\right)^{2} \otimes \psi_{s} \psi_{s}^{\prime}\right) .
$$

${ }^{32}$ The threshold that we use explicitly adjust for the temporal variation in the daily continuous volatility based on the realized bipower variation over the previous day as well as the strong intraday volatility pattern based on an estimate of the time-of-day effect; for additional details see Bollerslev and Todorov (2011b).
} 
risks are treated the same by investors. ${ }^{33}$

At a general level, the test therefore also supports the idea that $L J V_{t}$ affords a "cleaner" proxy for market fears than the variance risk premium, let alone the popularly used VIX "investor fear gauge." To further explore this, we turn next to the results from a series of standard monthly-based return predictability regressions for returns horizons ranging up to a year, using different variation measures as explanatory variables.

\section{Return Predictability Regressions}

Several recent studies have argued for the existence of a statistically significant link between the variance risk premium and future returns on the aggregate market portfolio, with this predictive relationship especially strong over 3-6 months horizons (see, e.g., Bollerslev, Tauchen, and Zhou, 2009; Drechsler and Yaron, 2011; Du and Kapadia, 2012; Bekaert and Hoerova, 2013; Bollerslev, Marrone, Xu, and Zhou, 2014; Camponovo, Scaillet, and Trojani, 2013; Vilkov and Xiao, 2013, among several other studies). The results from our predictability regressions complement and expand on these findings by explicitly considering the new jump tail variation measures, and the part of the variance risk premium due to jump tail risk, as separate predictor variables.

Following standard practice in the literature, we rely on a monthly observation frequency for all of our return predictability regressions. Specifically, let $r_{[t, t+\tau]}$ denote the continuously compounded return from time $t$ to $t+\tau$ formally defined in equation (2.2) above, with the unit time interval corresponding to a month. The return regressions discussed below may then be expressed as,

$$
r_{[t, t+h]}=a_{h}+b_{h} V_{t}+u_{t, t+h}, \quad t=1,2, \ldots, T-h,
$$

where $V_{t}$ refers to one or more of the variation measures and other explanatory variables, and the horizon range from $h=1$ (one-month) to $h=12$ (one year). To account for the overlap that occur for $h>1$, we rely on the standard robust Newey-West t-statistics with

\footnotetext{
${ }^{33}$ This particular choice of threshold results in a total of 285 left tail jumps over the full sample. We also experimented with other choices of $\eta_{s}$, resulting in equally strong rejections. The corresponding test for the right jump tails equals 65.0 , which also strongly rejects the null of identical jump tail "shapes" under the $\mathbb{P}$ and $\mathbb{Q}$ measures.
} 
a lag length equal to two times the return horizon. In addition, to help alleviate concerns about size distortions and over-rejections known to plague inference in overlapping return regressions with persistent predictor variables (see, e.g., Ang and Bekaert, 2007), following Hodrick (1992) we also report robust t-statistics for the null of no predictability from the reverse regressions. ${ }^{34}$ Further, to aid with the interpretation of the results from the multiple regressions involving more than one explanatory variable, we report Newey-West and Hodrick Wald-tests for the null of no predictability by any of the predictor variables included in the regression. ${ }^{35}$ We turn next to a discussion of the specific explanatory variables considered in the return predictability regressions.

\subsection{Variation Measures and Other Explanatory Variables}

Our estimation of the jump tail parameters $\hat{\alpha}_{t}^{ \pm}$and $\hat{\phi}_{t}^{ \pm}$, and in turn the new jump tail variation measures discussed above, closely follows the approach developed in Bollerslev and Todorov (2014) and the choice of a weekly estimation frequency advocated therein. Even though this result in approximately unbiased parameter estimates, the estimates are invariably somewhat noisy. To help smooth out this estimation error, and directly match the observation frequency of the jump tail variation measures to the observation frequency of the return regressions, in the empirical results reported on below we rely on the monthly variation measures obtained by averaging the within month weekly values. ${ }^{36}$ Comparing the plot for the resulting monthly $L J V_{t}$ series given in the top panel in Figure 5 to the weekly series shown in the top panel in Figure 2 clearly shows the effect of smoothing out the estimation error, and the implicit use of a coarser monthly estimation frequency. Still, the weekly and monthly series obviously share the same general features and dynamic dependencies.

The second panel in Figure 5 plots the monthly $V I X_{t}^{2}$ series. The $V I X$, of course, represents an approximation to the risk-neutral expectation of the total quadratic variation

\footnotetext{
${ }^{34}$ As forcefully emphasized by Hodrick (1992), the Hodrick-based t-statistics are only formally valid under the null of no predictability, not just by the regressor in question but by any predictor variable. As such, they are difficult to interpret even in simple regressions when more than one explanatory variable is useful for predicting the returns, let alone in multiple regressions. By contrast, the Newey-West t-statistics are always (asymptotically) justified and interpretable.

${ }^{35}$ With two predictor variables, the five- and one-percent critical values in the corresponding asymptotic Chi-square distribution with two degrees of freedom equal 5.991 and 9.210, respectively.

${ }^{36}$ For reasons of symmetry, we apply the same monthly averaging to all of the variation measures used as explanatory variables in the return regressions.
} 
in (2.3), and as such reflects the compensation for both time-varying diffusive volatility and jump intensity risks, as well as market expectations about future jump tail events and the "special" pricing thereof. Meanwhile, comparing our $L J V_{t}$ fear proxy to the $V I X_{t}^{2}$ reveals a strong coherence between the two series. At the same time, however, there are also some important differences. In particular, even though both series attained their maximum insample values around October 2008, the VIX fairly quickly reverted to pre-crisis levels, while $L J V_{t}$ remained elevated for a much longer period of time. $L J V_{t}$ also experienced another more prolonged period of elevated jump tail risk in 2010 in connection with the European sovereign debt crisis. Similarly, $L J V_{t}$ remained unusually high over a longer period of time than $V I X_{t}^{2}$ in the aftermath of the East Asian crises starting in July 1997 and the August 1998 Russian default. Conversely, the collapse of the tech bubble and the declining equity valuations in 2002 clearly resulted in higher values of the VIX, but hardly affect the left jump variation. ${ }^{37}$

The third panel in Figure 5 shows the variance risk premium minus the left jump tail variation $V R P_{t}-L J V_{t}$, or the part of the variance risk premium attributable to "normal" sized price fluctuations. ${ }^{38}$ Our estimate of the variance risk premium is based on the difference between the $V I X_{t}^{2}$ and the expectation of the forward variation aggregated over the month, as derived from the same multivariate forecasting model for the high-frequency based realized variation measures developed in Bollerslev and Todorov (2011b). ${ }^{39}$ Interestingly, the figure reveals a much less dramatic impact of the recent financial crisis on $V R P_{t}-L J V_{t}$ than on $L J V_{t}$ and $V I X_{t}^{2}$. On the other hand, $V R P_{t}-L J V_{t}$ was relatively high for part of 2002-03, while this period hardly registered for the left jump tail variation measure. These differences in the variation measures also directly manifest in the return predictability regressions discussed below.

As previously noted, the log dividend-price ratio arguable represents the most widely used

\footnotetext{
${ }^{37}$ These differences between the $V I X$ and $L J V$ mirror the differences between the option-based estimates for the time-varying diffusive and jump intensity risks in the fully parametric three-factor stochastic volatility model recently estimated by Andersen et al. (2013).

${ }^{38}$ Following a number of recent studies in the empirical asset pricing literature, we will refer to $-V R P_{t}$ as the variance risk premium, and correspondingly $V R P_{t}-L J V_{t}$ as the part of the premium due to "normal" sized price moves. This, of course, is immaterial for the fit of the return predictability regressions.

${ }^{39}$ Consistent with the idea that on average it is profitable to sell volatility, the sample mean of $V R P_{t}$ equals 1.20 in annualized percentage form.
} 
predictor variable in the literature. Although, it is commonly thought that the dividendprice ratio offers the most predictability over longer multi-year horizons, the results in Ang and Bekaert (2007) suggest that the predictability is actually the strongest over shorter within-year horizons as analyzed here. In parallel to the variation-based measures, the $\log$ dividend-price ratio $\log \left(D_{t} / P_{t}\right)$ shown in the bottom panel in Figure 5 also increased quite dramatically during the recent financial crisis and the accompanying precipitous drop in equity valuations. Meanwhile, comparing the plot of $\log \left(D_{t} / P_{t}\right)$ to the plots of the variationbased measures in the first three panels reveals a much more smoothly evolving series void of most of the other clearly discernible peaks associated with other readily identifiable economic and financial events. ${ }^{40}$

The next section presents the results from the return predictability regressions based on our new $L J V_{t}$ measure and these other explanatory variables. We begin by discussing the results for the aggregate market portfolio $M R K$.

\subsection{Aggregate Market Return}

As noted above, the predictability afforded by the variance risk premium appears to be the strongest over intermediate 3-6 months return horizons. Focussing on the 6-months horizon, the left panel in Table 2 reports the results from simple predictability regressions with standard Newey-West t-statistics in parentheses and Hodrick t-statistics in square brackets. The sixth column, in particular, confirms the existing empirical evidence that a higher (lower) variance risk premium tends to be associated with higher (lower) returns over the next six months. Meanwhile, comparing the results to the regression reported in the first column, the degree of predictability inherent in $V R P_{t}$ is dominated by that of the $L J V_{t}$ left jump tail variation measure. Further to this effect, subtracting $L J V_{t}$ from $V R P_{t}$ results in less significant t-statistics for $V R P_{t}-L J V_{t}$, and lowers the $R^{2}$ relative to the regression based on $V R P_{t}$ by a factor of a-half.

The regressions for the left jump variation measures $L J V_{t}^{*}$ and $L J V_{t}^{* *}$ that restrict either $\alpha_{t}^{-}$or $\phi_{t}^{-}$to be constant show that allowing for temporal variation in both the "shape" and

\footnotetext{
${ }^{40}$ The first order autocorrelation for each of the four monthly series shown in Figure 5 equal 0.67, 0.79, 0.53 , and 0.96 , respectively.
} 
the "level" of the jump tails enhances the predictability. Indeed, the regression coefficient associated with the $L J V_{t}^{*}$ jump tail variation measure extracted under the conventional assumption of time-invariant tail "shapes" is insignificant. ${ }^{41}$ Also, the right jump variation measure $R J V_{t}$ does not help in predicting the future returns.

The results from the multiple regressions reported in the right panel of the table further corroborates these findings. Including either $R J V_{t}$ or $V R P_{t}-L J V_{t}$ in a multiple regression together with $L J V_{t}$, leaves only $L J V_{t}$ significant. ${ }^{42}$ Also, even though the inclusion of $V I X_{t}^{2}$ in the multiple regression with $L J V_{t}$ renders the t-statistics for $L J V_{t}$ insignificant at the conventional five-percent level, the joint Wald-tests for the null of no predictability are overwhelmingly significant. Moreover the $R^{2}$ from the multiple regression based on $L J V_{t}$ and $V I X_{t}^{2}$ hardly increases relative to the $R^{2}$ from the simple regression based on $L J V_{t}$ only.

Altogether, this suggests that much of the return predictability previously ascribed to the variance risk premium is effectively coming from the part of the premium due to the left jump tail variation. Further along these lines, it is worth noting that replacing $L J V_{t}$ with the nonparametric kernel density estimate thereof discuss in Section 5.2, reduces the $R^{2}$ from 6.54 to 4.54 . Moreover, when including both the fitted value of $L J V_{t}$ and the corresponding residual from the nonparametric regression as explanatory variables in the same return predictability regression, both remain significant with Newey-West $t$-statistics of 2.17 and 2.13, respectively, underscoring the fact that $L J V_{t}$ and the predictability therein cannot be spanned by the market spot volatility.

As an additional robustness check, we also include the log dividend-price ratio as a possible predictor variable. In line with the existing literature (see, e.g., Ang and Bekaert, 2007 , and the many additional references therein), the t-statistics for $\log \left(D_{t} / P_{t}\right)$ in the simple regression reported in the last column in the first part of Table 2 are highly significant. Consistent with the results for the return horizons in excess of three months reported in

\footnotetext{
${ }^{41}$ The plots of $1 / \hat{\alpha}_{t}^{-}$and $L J I_{t}$ previously discussed in Figure 1 also suggest that most of the discernable variation in $L J V_{t}$ reside in the "shape" as opposed to the intensity of the "large" negative jumps.

${ }^{42}$ Including $L J V_{t}$ and $V R P_{t}$ in the same regression, obviously results in the same $R^{2}$ as the regressions based on $L J V_{t}$ and $V R P_{t}-L J V_{t}$. The Newey-West t-statistics for $V R P_{t}$ and $V R P_{t}-L J V_{t}$ are also the same, while the t-statistics for $L J V_{t}$ drops from 4.39 for the regression reported in Table 2 to 3.66 for the regression based on $L J V_{t}$ and $V R P_{t}$.
} 
Bollerslev, Tauchen, and Zhou (2009), the $R^{2}$ of 18.9 also far exceeds that of the variance risk premium and the other return variation measures. Meanwhile, including $\log \left(D_{t} / P_{t}\right)$ and $L J V_{t}$ in the same multiple regression further increases the $R^{2}$ to 21.4 , and even though the t-statistics for $\log \left(D_{t} / P_{t}\right)$ and $L J V_{t}$ are reduced somewhat compared to the two simple regressions, the Wald tests for their joint significance are highly significant. As such, this supports the idea that the dividend yield is not able to fully span the predictive information in the $L J V$ tail variation measure. This, of course, is also corroborated by the stylized theoretical equilibrium models discussed in Section 2.3.

Turning to Table 3 and the regression results for other return horizons reveal the same general patterns vis-a-vis the predictability in the variance risk premium and its jump tail component. In particular, while $V R P_{t}-L J V_{t}$ is significant in all of the simple regressions, except for the one-month horizon, the simple regressions based on $L J V_{t}$ generally results in larger t-statistics and the $R^{2}$ s are also much higher. ${ }^{43}$ The plots of the corresponding Newey-West $t$-statistics and adjusted regression $R^{2}$ s for all of the one through 12-months return regressions shown in Figure 6 further illustrate this. The $t$-statistics from the simple regressions based on $L J V_{t}$ are all significant, and the $R^{2}$ s increase with the return horizons. On the other hand, the $R^{2}$ s from the simple regressions based on $V R P_{t}-L J V_{t}$ plateaus at the four-month horizon, while the $R^{2}$ s from the multiple regressions based on both predictor variables are fairly close to those from the simple regressions based on $L J V_{t}$ only.

The general setup and discussion in Section 2, along with the test in Section 5.4, suggest that the $V R P_{t}-L J V_{t}$ predictor variable may be interpreted as a measure of economic uncertainty, while $L J V_{t}$ is more readily associated with notions of market sentiment, or fears. To further buttress this interpretation and help explain where the predictability is coming from, we turn next to a series of predictability regressions for various portfolio sorts and the three common Fama-French-Carhart risk factors.

\footnotetext{
${ }^{43}$ To put the monthly $R^{2}$ s reported in the table into perspective, Huang, Jiang, Tu, and Zhou (2013) have recently shown that a properly aligned version of the investor sentiment index originally proposed by Baker and Wurgler (2006) results in a statistically and economically significant predictive relationship for the monthly aggregate market returns with an $R^{2}$ of 1.54 percent.
} 


\subsection{Portfolio Sorts and Risk Factors}

Different portfolios may respond differently to changes in risk and risk aversion depending on their risk exposures. To this end, Table 4 reports the results from the same multiple regressions based on $L J V_{t}$ and $V R P_{t}-L J V_{t}$ reported in Tables 2 and 3, replacing the aggregate market portfolio with portfolios comprised of stocks sorted according to their market capitalization, book-to-market value, and most recent annual return. For considerations of space, we focus our discussion on the six equally weighted portfolios made up of the top and bottom quintiles for each of the three different sorts.

Beginning with the results pertaining to the size-sorted portfolios reported in the first two columns of Table 4, a clear distinction emerges in the influences of $L J V_{t}$ and $V R P_{t}-L J V_{t}$. In particular, while $V R P_{t}-L J V_{t}$ is not as significant as $L J V_{t}$ for the aggregate market portfolio, it is the most significant predictor for the small-stock portfolio. The regression $R^{2} \mathrm{~s}$ for the small-stock portfolio reach an impressive 19.3 percent at the annual level. Meanwhile, the results for the large-stock portfolio more closely mirror those for the aggregate market, with the predictability is primarily attributable to $L J V_{t}$.

To purge the returns from systematic market risks, the third column reports the regression results from the Small Minus Big $(S M B)$ zero-cost portfolio defined by the returns on the previously discussed small minus large-stock portfolios. In contrast to the aggregate market portfolio, the longer-run predictability for this portfolio is exclusively coming from the $V R P_{t}-L J V_{t}$ variation measure. The results from the simple regressions for the $S M B$ portfolio shown in Figure 7 further underscore this point. While the $t$-statistics associated with $V R P_{t}-L J V_{t}$ for predicting the three through 12-months returns are all significant at conventional levels, the $t$-statistics for $L J V_{t}$ are all insignificant. Moreover, the $R^{2}$ s from the multiple regressions that include both $V R P_{t}-L J V_{t}$ and $L J V_{t}$, shown in the bottom panel of the figure, are very close to the $R^{2}$ s from the simple regressions based on $V R P_{t}-L J V_{t}$ only.

The $S M B$ portfolio has previously been associated with variables that describe changes in the investment opportunity set (e.g., Petkova, 2006). As such, our finding that the predictability of the $S M B$ portfolio is solely driven by $V R P_{t}-L J V_{t}$ is consistent with the idea 
that this measure is most directly associated with notions of economic uncertainty. Further along these lines, a number of studies have argued that smaller firms tend to be more strongly affected by credit market conditions than larger firms and therefore also more susceptible to general economic conditions (see, e.g., Perez-Quiros and Timmermann, 2000), thus helping to explain why $V R P_{t}-L J V_{t}$ is a better predictor for the return on the small-stock portfolio.

The results for the value and growth portfolios along with the corresponding High Minus Low $(H M L)$ book-to-market portfolio reported in the next three columns in Table 4 convey a very different picture. The predictability pattern for the low book-to-market portfolio, in particular, fairly closely mirror that of the aggregate market portfolio. On the other hand, the $R^{2}$ s for the $H M L$ portfolio in Figure 8 appear to be maximized at the intermediate four months horizon, with all of the predictability attributable to the $L J V_{t}$ jump tail variation measure.

The returns on portfolios comprised of growth stocks have previously been related to measures of funding liquidity risk (e.g., Asness et al., 2013). Thus, to the extend that liquidity conditions reflect market sentiment, the results in Figure 8 for the the $H M L$ portfolio again corroborate the interpretation of $L J V_{t}$ as a measure of market sentiment, or fears.

Turning to the results for the momentum portfolios, reported in the last three columns in Table 4, reveal some very high $R^{2}$ s for the "loser" portfolio made up of the stocks that performed the worst over the previous 12 months. As shown in Figure 9, this also translates into very impressive $R^{2}$ s for the Winners Minus Losers $(W M L)$ portfolio in excess of thirty percent at the 6-8 months horizons. In contrast to the predictability of the $S M B$ portfolio, which appears to be driven solely by $V R P_{t}-L J V_{t}$, and the predictability of $H M L$, which is solely attributable to $L J V_{t}$, both of the two variation measures contribute to the predictability of the $W M L$ portfolio.

The return on momentum portfolios, and "loser" portfolios in particular, have previously been associated with funding liquidity risk (e.g., Pastor and Stambaugh, 2003; Korajczyk and Sadka, 2004). The returns on momentum portfolios have also been shown to exhibit option like characteristics and be predictable by the "state" of the economy and the volatility of the aggregate market (e.g., Daniel et al., 2012; Daniel and Moskowitz, 2013), as well as the magnitude of the volatility risk premium (Nagel, 2012; Fan et al., 2013, e.g.,). Consis- 
tent with these earlier findings, the much stronger predictability for the "loser" and $W M L$ portfolio based on the decomposition of $V R P_{t}$ reported here again support the interpretation of $V R P_{t}-L J V_{t}$ and $L J V_{t}$ as separate proxies for market uncertainty and sentiment, respectively, both of which help predict the momentum returns.

\section{Conclusion}

The variance risk premium, defined as the difference between the actual and risk-neutralized expectations of the forward variation of the aggregate market portfolio, is naturally decomposed into two fundamentally different sources of market variance risk: "normal" sized price fluctuations and jump tail risk. We argue that the part of the variance risk premium associated with the compensation for left jump tail risk, in particular, may be interpreted as a measure for market sentiments, or fears. We develop new procedures for empirically estimating this component of the variance risk premium from the options surface at a given point in time. Consistent with the idea that this jump risk component represents separate state variables that drive the market risk premium, we find that the explanatory power provided by return predictability regressions based on the total variance risk premium, as previously reported in the literature, may be significantly enhanced by including the jump tail risk component as a separate predictor variable. Our empirical findings corroborate the theoretical equilibrium-based interpretation of the jump tail risk component as being more closely associated with changes in risk aversion, as opposed to changes in market risks. The predictability patterns observed for other commonly studied portfolio sorts and systematic risk factors convey a similar message: the predictability of the approximately market-neutral small-minus-big portfolio appears to be driven solely by the non-jump component, the highminus-low book-to-market portfolio is only predicted by the jump risk component, while both components are highly significant for predicting the winners-minus-losers portfolio returns.

Our new jump tail risk measure is obviously related to the pricing of tail risk in the economy and investors' attitude toward risk more generally. It would be interesting to more fully explore these relations, and how the new measure relates to other directly observable economic variables and market-based sentiment indicators. Further along these lines, while the theoretical arguments underlying our interpretation of the variance risk premium 
components and the econometric procedures underlying their separate estimation are both essentially model-free, a more structural-based estimation might afford a deeper understanding of the economic mechanisms that drive the temporal variation in the separate components and help shed new light on the validity of competing equilibrium-based asset pricing models. We leave further work along these lines for future research. 


\section{References}

Almeida, C., J. Vicente, and O. Guillen (2013). Nonparametric Tail Risk and Stock Returns: Predictability and Risk Premia. Getulio Vargas Foundation, Rio de Janeiro, Working paper.

Andersen, T. G., T. Bollerslev, P. C. Christoffersen, and F. X. Diebold (2013). Finacial Risk Measurement for Financial Risk Management. In M. H. G. Constantinides and R. Stulz (Eds.), Handbook of the Economics of Finance. Elsevier Inc.

Andersen, T. G., N. Fusari, and V. Todorov (2013). The Risk Premia Embeddded in Index Options. Northwestern University, Working paper.

Andreou, E. and E. Ghysels (2013). What Drives the VIX and the Volatility Risk Premium? University of North Carolina, Working paper.

Ang, A. and G. Bekaert (2007). Stock Return Predictability: Is it There? Review of Financial Studies 20, 651-707.

Asness, C. S., T. Moskowitz, and L. H. Pedersen (2013). Value and Momemtum Everywhere. Journal of Finance 68, 929-985.

Baker, M. and J. Wurgler (2006). Investor Sentiment and the Cross-section of Stock Returns. Journal of Finance 61, 1645-1680.

Bakshi, G. and N. Kapadia (2003). Delta-Hedge Gains and the Negative Market Volatility Risk Premium. Review of Financial Studies 16, 527-566.

Bakshi, G. and D. Madan (2006). A Theory of Volatility Spreads. Management Science 52, 19451956.

Bali, T. G. and H. Zhou (2013). Risk, Uncertainty, and Expected Returns. Georgetown University, Working paper.

Bansal, R. and I. Shaliastovich (2011). Learning and Asset-Price Jumps. Review of Financial Studies 24, 2738-2780.

Bansal, R. and A. Yaron (2004). Risks for the Long Run: A Potential Resolution of Asset Pricing Puzzles. Journal of Finance 59, 1481-1509.

Bates, D. S. (2000). Post-'87 Crash Fears in S\&P 500 Future Options. Journal of Econometrics 94, $181-238$.

Bekaert, G. and E. Engstrom (2010). Asset Return Dynamics under Bad Environment - Good Evironment Fundamentals. Columbia University, Working paper.

Bekaert, G. and M. Hoerova (2013). The VIX, the Varince Premium and Stock Market Volatility. Columbia University, Working paper.

Bekaert, G., M. Hoerova, and M. Lo Duca (2012). Risk, Uncertainty and Monetary Policy. Columbia University, Working paper. 
Bollerslev, T., J. Marrone, L. Xu, and H. Zhou (2014). Stock Return Predictability and Variance Risk Premia: Statistical Inference and International Evidence. Journal of Financial and Quantitative Analysis. Forthcoming.

Bollerslev, T., N. Sizova, and G. Tauchen (2012). Volatility in Equilibrium: Asymmetries and Dynamic Dependencies. Review of Finance 16, 31-80.

Bollerslev, T., G. Tauchen, and H. Zhou (2009). Expected Stock Returns and Variance Risk Premia. Review of Financial Studies 22, 4463-4492.

Bollerslev, T. and V. Todorov (2011a). Estimation of Jump Tails. Econometrica 79, 1727-1783.

Bollerslev, T. and V. Todorov (2011b). Tails, Fears and Risk Premia. Journal of Finance 66, $2165-2211$.

Bollerslev, T. and V. Todorov (2014). Time-Varying Jump Tails. Journal of Econometrics. Forthcoming.

Bondarenko, O. (2014). Variance Trading and Market Price of Variance Risk. Journal of Econometrics 180, 81-97.

Bowman, A. and A. Azzalini (1997). Applied Smoothing Techniques for Data Analysis. Oxford University Press.

Broadie, M., M. Chernov, and M. Johannes (2007). Specification and Risk Premiums: The Information in S\&P 500 Futures Options. Journal of Finance 62, 1453-1490.

Campbell, J. and J. Cochrane (1999). By Force of Habit: A Consumption Based Explanation of Aggregate Stock Market Behavior. Journal of Political Economy 10\%, 205-251.

Camponovo, L., O. Scaillet, and F. Trojani (2013). Predictive Regression and Robust Hypothesis Testing: Predictability Hidden by Anomalous Observations. University of Lugano, Working paper.

Carr, P., H. Geman, D. Madan, and M. Yor (2003). Stochastic Volatility for Lévy Processes. Mathematical Finance 13, 345-382.

Carr, P. and L. Wu (2009). Variance Risk Premiums. Review of Financial Studies 22, 1311-1341.

Chollete, L. and C. Lu (2011). The Market Premium for Dynamic Tail Risk. University of Stavanger, Norway, Working paper.

Christoffersen, P., S. Heston, and K. Jacobs (2009). The Shape and Term Structure of the Index Option Smirk: Why Multifactor Stochastic Volatility Models Work so Well. Management Science 55, 1914-1932.

Christoffersen, P., K. Jacobs, and C. Ornthanalai (2012). Dynamic Jump Intensities and Risk Premiums: Evidence from S\&P 500 Returns and Options. Journal of Financial Economics 106, $447-472$.

Daniel, K., R. Jagannathan, and S. Kim (2012). Tail Risk in Momentum Strategy Returns. University of Chicago and Northwestern University, Working paper. 
Daniel, K. and T. Moskowitz (2013). Momentum Crashes. University of Chicago and Columbia University, Working paper.

Davis, R. and T. Mikosch (2009). The Extremogram: A Correlogram for Extreme Events. Bernoulli 15, 977-1009.

Davis, R., T. Mikosch, and I. Cribben (2012). Towards, Estimating Extremal Serial Dependence via the Bootstrapped Extremogram. Journal of Econometrics 170, 142-152.

Drechsler, I. and A. Yaron (2011). What's Vol Got to Do with It? Review of Financial Studies 24, $1-45$.

Du, D. (2010). General Equilibrium Pricing of Options with Habit Formation and Event Risks. Journal of Financial Economics 99, 400-426.

Du, J. and N. Kapadia (2012). Tail and Volatility Indices from Option Prices. University of Massachusetts, Amhurst, Working paper.

Duffie, D. (2001). Dynamic Asset Pricing Theory (3rd ed.). Princeton University Press.

Duffie, D., J. Pan, and K. Singleton (2000). Transform Analysis and Asset Pricing for Affine Jump-Diffusions. Econometrica 68, 1343-1376.

Eraker, B. (2004). Do Stock Prices and Volatility Jump? Reconciling Evidence from Spot and Option Prices. Journal of Finance 59, 1367-1403.

Eraker, B. and I. Shaliastovich (2008). An Equilibrium Guide to Designing Affine Pricing Models. Mathematical Finance 18, 519-543.

Eraker, B. and J. Wang (2012). A Non-Lineaer Dynamic Model of the Variance Risk Premium. University of Wisconsin, Working paper.

Fan, J., M. B. Imerman, and W. Dai (2013). What Does the Volatility Risk Premium Say About Liquidity Provision and Demand for Hedging Tail Risk? Princeton University, Working paper.

Gabaix, X. (2012). Variable Rare Disasters: An Exactly Solved Framework for Ten Puzzles in Macro-Finance. Quarterly Journal of Economics 127, 645-700.

Hamidieh, K. (2011). Estimating the Tail Shape Parameter from Option Prices. California State University Fullerton, Working paper.

Han, B. and Y. Zhou (2011). Variance Risk Premium and Cross-Section of Stock Returns. University of Texas at Austin, Working paper.

Hodrick, R. J. (1992). Dividend Yields and Expected Stock Returns: Alternative Procedures for Inference and Measurement. Review of Financial Studies 5, 357-368.

Huang, D., F. Jiang, J. Tu, and G. Zhou (2013). Investor Sentiment Aligned: A Powerful Predictor of Stock Returns. Washington University St.Louis, Working paper.

Kelly, B. and H. Jiang (2013). Tail Risk and Asset Prices. University of Chicago, Working paper. 
Korajczyk, R. and R. Sadka (2004). Are Momentum Profits Robust to Trading Costs? Journal of Finance 59, 1039-1082.

Li, J. and G. Zinna (2014). Variance Components, Term Structure of Variance Risk Premia, and Expected Asset Returns. ESSEC Business School, Working paper.

Mancini, C. (2001). Disentangling the Jumps of the Diffusion in a Geometric Brownian Motion. Giornale dell'Istituto Italiano degi Attuari LXIV, 19-47.

Merton, R. (1973). An Intertemporal Capital Asset Pricing Model. Econometrica 41, 867-887.

Nagel, S. (2012). Evaporating Liquidity. Review of Financial Studies 25, 2005-2039.

Pan, J. (2002). The Jump-Risk Premia Implicit in Options: Evidence from an Integrated TimeSeries Study. Journal of Financial Economics 63, 3-50.

Pastor, L. and R. F. Stambaugh (2003). Liquidity Risk and Expected Stock Returns. Journal of Political Economy 111, 642-685.

Perez-Quiros, P. and A. Timmermann (2000). Firm Size and Cyclical Variations in Stock Returns. Journal of Finance 55, 1229-1262.

Petkova, R. (2006). Do the Fama-French Factors Proxy for Innovatins in Predictive Variables. Journal of Finance 61, 581-612.

Protter, P. (2004). Stochastic Integration and Differential Equations (2nd ed.). Berlin: SpringerVerlag.

Ruenzi, S. and F. Weigert (2011). Crash Sensitivity and the Cross-Section of Expected Stock Returns. University of Mannheim, Working paper.

Santa-Clara, P. and S. Yan (2010). Crashes, Volatility, and the Equity Premium: Lessons from S\&P 500 Options. Review of Economics and Statistics 92, 435-451.

Schneider, P. (2012). Fear Trading. University of Lugano, Working paper.

Schwert, G. W. (2011). Stock Volatility during the Recent Financial Crisis. European Financial Management 17, 789-805.

Siriwardane, E. (2013). The Probability of Rare Disasters: Estimation and Implications. NYU Stern School of Business, Working paper.

Todorov, V. (2010). Variance Risk Premia Dynamics: The Role of Jumps. Review of Financial Studies 23, 345-383.

Vilkov, K. and Y. Xiao (2013). Option-Implied Information and Predictability of Extreme Returns. Goethe University Frankfurt, Working paper.

Wachter, J. A. (2013). Can Time-Varying Risk of Rare Disasters Explain Aggregate Stock Market Volatility? Journal of Finance 68, 987-1035. 


\section{Table 1: Summary Statistics}

The table reports summary statistics for weekly returns, estimated jump tail parameters, and jump tail variation measures. The data ranges from January 1996 to August 2013. The variation measures are recorded at the end-of-the-week, with the returns spanning the corresponding Monday close-to-close. The returns are in weekly percentage form. All of the variation measures are in annualized percentage form, except for the right tail variation measure which is in annualized percentage square form.

Panel A: Univariate Statistics

\begin{tabular}{c|cccc|cccc|cccc}
\hline \hline & MRK & SML & HML & WML & $\alpha^{-}$ & $\alpha^{+}$ & LJI & RJI & LJV & RJV & LJV $^{*}$ & LJV $^{* *}$ \\
\hline Mean & 0.14 & 0.03 & 0.06 & 0.21 & 16.23 & 61.81 & 3.61 & 0.55 & 0.45 & 0.02 & 0.37 & 0.52 \\
Std. Dev & 2.89 & 2.15 & 2.50 & 4.83 & 5.33 & 19.68 & 3.19 & 2.04 & 0.54 & 0.05 & 0.23 & 0.72 \\
Skewness & -0.33 & -0.83 & 0.11 & -0.95 & 0.43 & 0.65 & 1.94 & 6.10 & 5.41 & 5.03 & 2.21 & 6.02 \\
Kurtosis & 6.35 & 10.32 & 5.24 & 11.48 & 3.00 & 3.52 & 8.90 & 44.99 & 48.62 & 32.80 & 12.04 & 63.49 \\
Max & 14.12 & 9.98 & 12.07 & 28.76 & 33.57 & 145.92 & 28.32 & 18.68 & 7.20 & 0.46 & 2.14 & 10.40 \\
Min & -15.06 & -14.83 & -11.33 & -34.40 & 3.87 & 18.93 & 0.02 & 0.00 & 0.01 & 0.00 & 0.00 & 0.00 \\
AR(1) & -0.16 & 0.07 & -0.10 & -0.03 & 0.59 & 0.67 & 0.24 & 0.07 & 0.69 & 0.11 & 0.28 & 0.52 \\
\hline
\end{tabular}

Panel B: Contemporaneous Correlations

\begin{tabular}{|c|c|c|c|c|c|c|c|c|c|c|c|c|}
\hline & MRK & SML & HML & WML & $\alpha^{-}$ & $\alpha^{+}$ & LJI & RJI & LJV & RJV & LJV $^{*}$ & $\mathrm{LJV}^{* *}$ \\
\hline MRK & 1.00 & -0.15 & 0.07 & -0.25 & -0.02 & 0.01 & -0.16 & -0.02 & -0.23 & -0.05 & -0.26 & -0.11 \\
\hline SML & & 1.00 & 0.36 & 0.00 & 0.05 & 0.04 & -0.03 & 0.00 & -0.09 & -0.01 & -0.03 & -0.02 \\
\hline HML & & & 1.00 & -0.32 & 0.03 & 0.06 & 0.01 & 0.01 & -0.13 & -0.02 & -0.03 & -0.06 \\
\hline WML & & & & 1.00 & 0.05 & 0.05 & 0.01 & -0.02 & 0.06 & 0.00 & 0.06 & 0.05 \\
\hline$\alpha^{-}$ & & & & & 1.00 & 0.68 & 0.17 & 0.17 & -0.53 & 0.12 & -0.00 & -0.54 \\
\hline$\alpha^{+}$ & & & & & & 1.00 & 0.42 & 0.10 & -0.35 & -0.01 & 0.12 & -0.25 \\
\hline LJI & & & & & & & 1.00 & 0.45 & 0.26 & 0.44 & 0.71 & 0.21 \\
\hline RJI & & & & & & & & 1.00 & -0.02 & 0.89 & 0.10 & 0.04 \\
\hline LJV & & & & & & & & & 1.00 & 0.04 & 0.42 & 0.72 \\
\hline RJV & & & & & & & & & & 1.00 & 0.19 & 0.06 \\
\hline LJV* & & & & & & & & & & & 1.00 & 0.18 \\
\hline LJVV* & & & & & & & & & & & & 1.00 \\
\hline
\end{tabular}

Panel C: One-Week-Ahead Return Correlations

\begin{tabular}{c|cccc|cccc}
\hline \hline & $\alpha^{-}$ & $\alpha^{+}$ & LJI & RJI & LJV & RJV & LJV $^{*}$ & LJV $^{* *}$ \\
\hline MRK & -0.01 & -0.03 & 0.02 & -0.01 & 0.05 & -0.00 & 0.06 & 0.01 \\
SML & & -0.00 & -0.05 & -0.02 & -0.12 & -0.03 & -0.09 & -0.06 \\
HML & & & -0.02 & 0.03 & -0.05 & 0.06 & -0.05 & -0.01 \\
WML & & & & -0.00 & -0.02 & -0.01 & 0.06 & -0.04 \\
\hline
\end{tabular}




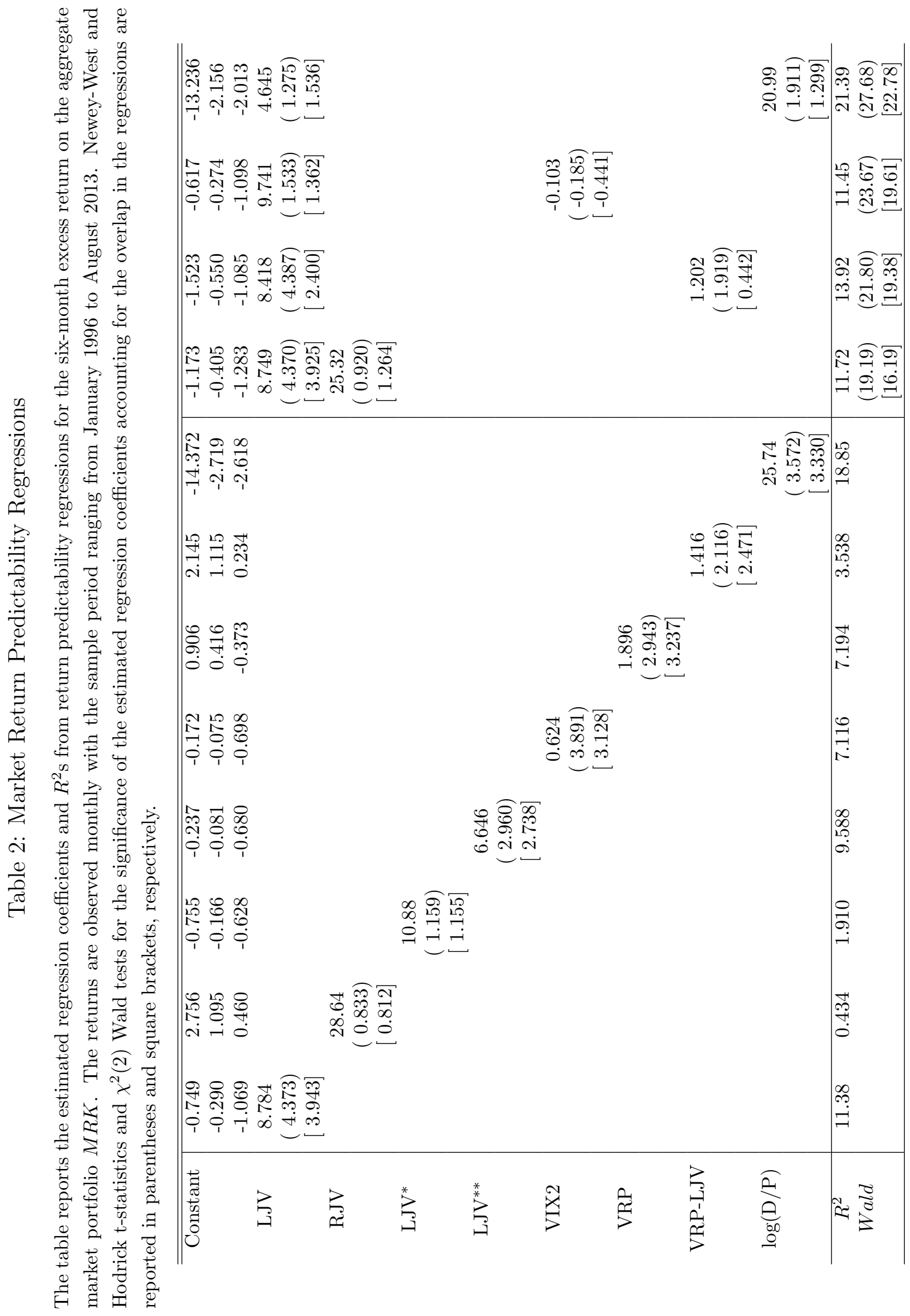




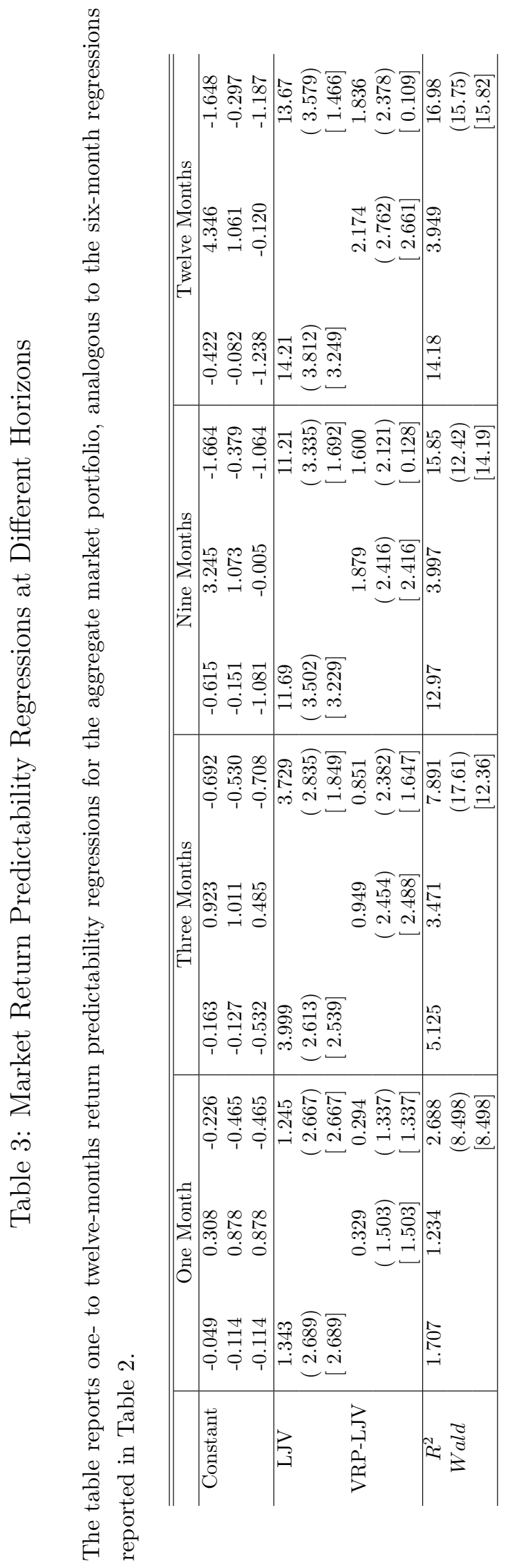




\section{Table 4: Portfolio Return Predictability Regressions}

The table reports the results from one- to twelve-months return predictability regressions for size (twenty percent smallest and biggest firms), book-to-market (twenty percent highest and lowest B/M ratios), and momentum (twenty percent top and bottom performers over the past 2-12 months) portfolios, along with the corresponding zero-cost portfolios, analogous to the results for the aggregate market in Tables 2 and 3.

\begin{tabular}{|c|c|c|c|c|c|c|c|c|c|}
\hline & Small & Big & SMB & High & Low & "HML & Winners & Losers & WML \\
\hline \multicolumn{10}{|c|}{ One Month Returns } \\
\hline \multirow[t]{3}{*}{ Constant } & 0.569 & 0.012 & 0.557 & 0.601 & -0.180 & 0.781 & 1.132 & -2.278 & 3.410 \\
\hline & $(0.980)$ & $(0.024)$ & $(1.462)$ & $(1.126)$ & $(-0.368)$ & $(2.395)$ & $(1.163)$ & $(-1.273)$ & $(2.369)$ \\
\hline & {$[0.980]$} & {$[0.024]$} & {$[1.462]$} & {$[1.126]$} & {$[-0.368]$} & {$[2.395]$} & [ 1.163$]$ & $-1.273]$ & [ 2.369] \\
\hline \multirow[t]{3}{*}{ LJV } & 0.027 & 1.179 & -1.151 & 0.260 & 1.430 & -1.169 & 1.281 & 4.327 & -3.045 \\
\hline & $(0.037)$ & $(2.666)$ & $(-2.040)$ & $(0.463)$ & $(3.244)$ & $(-2.761)$ & $(1.337)$ & $(1.606)$ & $(-1.189)$ \\
\hline & {$[0.037]$} & {$[2.666]$} & {$[-2.040]$} & {$[0.463]$} & {$[3.244]$} & {$[-2.761]$} & [ 1.337$]$ & {$[1.606]$} & {$[-1.189]$} \\
\hline \multirow[t]{3}{*}{ VRP-LJV } & 0.411 & 0.252 & 0.159 & 0.303 & 0.377 & -0.074 & 0.270 & 1.817 & -1.547 \\
\hline & $(1.744)$ & $(1.163)$ & $(0.960)$ & $(1.321)$ & $(1.792)$ & $(-0.425)$ & $(0.601)$ & $(2.060)$ & $(-1.692)$ \\
\hline & {$[1.744]$} & [ 1.163$]$ & {$[0.960]$} & {$[1.321]$} & [ 1.792$]$ & $-0.425]$ & {$[0.601]$} & {$[2.060]$} & {$[-1.692]$} \\
\hline$R^{2}$ & 1.134 & 2.449 & 1.780 & 0.900 & 3.867 & 2.551 & 0.521 & 4.518 & 4.156 \\
\hline \multirow[t]{2}{*}{ Wald } & $(3.042)$ & $(7.795)$ & $(7.592)$ & $(1.865)$ & $(13.73)$ & $(8.388)$ & $(1.842)$ & $(5.066)$ & $(2.871)$ \\
\hline & {$[3.042]$} & [7.795] & {$[7.592]$} & {$[1.865]$} & {$[13.73]$} & {$[8.388]$} & {$[1.842]$} & {$[5.066]$} & {$[2.871]$} \\
\hline \multicolumn{10}{|c|}{ Three Months Returns } \\
\hline \multirow[t]{3}{*}{ Constant } & 0.546 & 0.174 & 0.373 & 1.459 & -0.548 & 2.008 & 3.656 & -10.59 & 14.25 \\
\hline & $(0.362)$ & $(0.133)$ & $(0.381)$ & $(1.001)$ & $(-0.420)$ & $(1.918)$ & $(1.448)$ & $(-2.019)$ & $(3.389)$ \\
\hline & {$[0.144]$} & {$[-0.141]$} & {$[0.451]$} & {$[0.765]$} & {$[-0.724]$} & {$[2.106]$} & {$[1.054]$} & {$[-2.003]$} & {$[3.137]$} \\
\hline \multirow[t]{3}{*}{ LJV } & 2.473 & 3.248 & -0.776 & 1.366 & 4.880 & -3.514 & 3.317 & 19.46 & -16.14 \\
\hline & $(1.686)$ & $(2.358)$ & $(-0.567)$ & ( 1.089$)$ & $(3.412)$ & $(-2.423)$ & $(0.953)$ & $(3.534)$ & $(-2.409)$ \\
\hline & {$[0.723]$} & [ 1.623] & {$[-1.015]$} & {$[0.278]$} & {$[2.650]$} & {$[-2.709]$} & {$[0.719]$} & [ 2.559] & {$[-2.041]$} \\
\hline \multirow[t]{3}{*}{ VRP-LJV } & 1.277 & 0.703 & 0.574 & 0.987 & 0.723 & 0.264 & 0.690 & 6.232 & -5.542 \\
\hline & $(2.710)$ & $(2.108)$ & $(2.311)$ & $(2.451)$ & $(2.079)$ & $(1.032)$ & $(1.069)$ & $(2.554)$ & $(-2.275)$ \\
\hline & {$[2.428]$} & {$[1.440]$} & {$[2.598]$} & [ 2.166] & [ 1.139$]$ & {$[1.594]$} & {$[0.756]$} & [ 2.359] & {$[-2.323]$} \\
\hline$R^{2}$ & 4.644 & 6.374 & 1.531 & 3.420 & 9.862 & 6.477 & 1.183 & 19.93 & 22.78 \\
\hline \multirow[t]{2}{*}{ Wald } & $(13.59)$ & $(11.11)$ & $(7.248)$ & $(6.371)$ & (19.39) & $(11.38)$ & $(1.784)$ & $(13.63)$ & $(6.129)$ \\
\hline & [13.30] & [8.299] & {$[6.886]$} & [6.023] & [14.19] & {$[8.387]$} & [1.497] & [13.46] & {$[6.701]$} \\
\hline \multicolumn{10}{|c|}{ Six Months Returns } \\
\hline Constant & -0.339 & 0.357 & -0.696 & 2.078 & -0.522 & 2.600 & 5.871 & -23.27 & 29.14 \\
\hline & $(-0.112)$ & $(0.127)$ & $(-0.386)$ & $(0.614)$ & $(-0.200)$ & $(1.127)$ & $(1.222)$ & $(-2.085)$ & $(3.660)$ \\
\hline & {$[-0.516]$} & {$[-0.522]$} & {$[-0.164]$} & {$[0.188]$} & {$[-0.993]$} & {$[1.367]$} & {$[0.555]$} & {$[-2.189]$} & {$[2.902]$} \\
\hline LJV & 7.697 & 7.285 & 0.412 & 5.339 & 9.196 & -3.857 & 9.253 & 50.34 & -41.09 \\
\hline & $(2.663)$ & ( 3.708$)$ & $(0.167)$ & $(1.772)$ & $(4.723)$ & $(-1.303)$ & $(1.833)$ & ( 3.669$)$ & $(-2.587)$ \\
\hline & {$[0.940]$} & [ 2.323] & [ - -1.238] & [ 0.991] & {$[2.762]$} & {$[-2.046]$} & [ 1.104$]$ & {$[2.420]$} & {$[-1.894]$} \\
\hline VRP-LJV & 2.437 & 0.861 & 1.576 & 1.462 & 0.917 & 0.545 & 1.536 & 7.693 & -6.157 \\
\hline & $(2.970)$ & $(1.419)$ & $(3.474)$ & $(1.673)$ & $(1.569)$ & $(0.969)$ & $(1.486)$ & $(2.484)$ & $(-2.401)$ \\
\hline & {$[2.004]$} & $0.145]$ & {$[3.879]$} & $1.337]$ & {$[-0.093]$} & [ 1.668$]$ & [0.429] & {$[0.771]$} & {$[-0.831]$} \\
\hline$R^{2}$ & 12.93 & 10.33 & 5.835 & 6.651 & 14.34 & 3.836 & 3.850 & 32.93 & 34.22 \\
\hline Wald & $(17.76)$ & (14.40) & (12.38) & $(3.732)$ & $(24.52)$ & $(7.408)$ & $(6.223)$ & (13.96) & (7.439) \\
\hline & [17.76] & [12.89] & [16.65] & {$[3.732]$} & [21.90] & [5.839] & [4.639] & {$[15.17]$} & [9.702] \\
\hline & & & & Nine Mor & is Return & & & & \\
\hline Constant & 0.015 & 1.107 & -1.092 & 3.275 & 0.137 & 3.138 & 8.196 & -27.825 & 36.021 \\
\hline & $(0.003)$ & $(0.240)$ & $(-0.368)$ & $(0.617)$ & $(0.032)$ & $(0.877)$ & $(1.030)$ & $(-1.903)$ & $(4.291)$ \\
\hline & {$[-0.512]$} & {$[-0.605]$} & $-0.076]$ & {$[0.016]$} & {$[-0.963]$} & [ 1.155$]$ & {$[0.185]$} & {$[-1.951]$} & [ 2.798] \\
\hline LJV & 11.20 & 9.582 & 1.621 & 8.055 & 11.48 & -3.421 & 13.96 & 63.60 & -49.64 \\
\hline & $(2.182)$ & $(2.975)$ & $(0.561)$ & $(1.382)$ & $(3.912)$ & $(-0.806)$ & $(2.703)$ & $(3.364)$ & $(-2.862)$ \\
\hline & [ 0.649$]$ & [ 1.759$]$ & {$[-1.083]$} & [ 0.793$]$ & {$[1.956]$} & {$[-1.875]$} & {$[1.200]$} & [ 1.767$]$ & {$[-1.707]$} \\
\hline VRP-LJV & 2.976 & 1.111 & 1.865 & 1.755 & 1.335 & 0.421 & 2.581 & 8.964 & -6.383 \\
\hline & $(2.802)$ & ( 1.446$)$ & $(2.506)$ & ( 1.658$)$ & ( 1.959$)$ & $(0.573)$ & ( 2.013$)$ & ( 2.701) & $(-2.402)$ \\
\hline & {$[1.082]$} & $-0.129]$ & $2.586]$ & $0.770]$ & $-0.141]$ & [ 1.485$]$ & {$[0.479]$} & $0.085]$ & {$[0.093]$} \\
\hline$R^{2}$ & 16.07 & 11.01 & 5.620 & 8.480 & 14.726 & 1.777 & 6.116 & 33.26 & 29.55 \\
\hline Wald & (15.03) & $(8.862)$ & $(8.707)$ & $(2.897)$ & (16.06) & (6.497) & (10.11) & $(13.40)$ & (8.979) \\
\hline & {$[10.26]$} & {$[10.06]$} & [8.404] & {$[2.516]$} & [19.85] & [7.204] & [9.543] & [13.25] & {$[9.221]$} \\
\hline & & & & velve $\mathrm{Mc}$ & hs Retur & & & & \\
\hline Constant & 0.872 & 2.020 & -1.148 & 5.120 & 0.818 & 4.303 & 10.76 & -28.06 & 38.83 \\
\hline & $(0.171)$ & $(0.339)$ & $(-0.261)$ & $(0.812)$ & $(0.145)$ & $(0.924)$ & $(1.029)$ & $(-1.752)$ & $(4.902)$ \\
\hline & {$[-0.458]$} & $-0.799]$ & {$[0.352]$} & $-0.140]$ & {$[-1.110]$} & {$[1.002]$} & {$[-0.121]$} & {$[-1.742]$} & [ 2.489] \\
\hline LJV & 13.18 & 11.61 & 1.577 & 9.995 & 13.86 & -3.860 & 18.99 & 68.61 & -49.63 \\
\hline & $(2.245)$ & $(3.122)$ & $(0.458)$ & ( 1.399$)$ & $(4.325)$ & $(-0.675)$ & $(2.989)$ & $(3.449)$ & $(-2.848)$ \\
\hline & {$[0.428]$} & {$[1.547]$} & {$[-1.754]$} & [ 0.822$]$ & {$[1.744]$} & {$[-1.054]$} & [ 1.318$]$ & [ 1.421$]$ & {$[-1.387]$} \\
\hline VRP-LJV & 3.714 & 1.147 & 2.566 & 1.450 & 1.571 & -0.121 & 2.953 & 9.317 & -6.364 \\
\hline & $(3.222)$ & $(1.382)$ & $(2.817)$ & ( 1.341$)$ & $(2.012)$ & $(-0.120)$ & ( 1.928$)$ & ( 3.089$)$ & $(-2.399)$ \\
\hline & {$[0.967]$} & {$[-0.354]$} & [ 3.675] & {$[-0.028]$} & {$[-0.300]$} & {$[0.871]$} & {$[0.061]$} & {$[-0.159]$} & [ 0.249$]$ \\
\hline$R^{2}$ & 19.31 & 11.16 & 7.616 & 8.101 & 15.30 & 1.537 & 7.629 & 30.30 & 22.72 \\
\hline Wald & $(26.43)$ & $(9.768)$ & (12.03) & $(2.580)$ & (18.98) & $(0.839)$ & (10.30) & $(18.33)$ & $(9.889)$ \\
\hline & [9.841] & [11.89] & [16.83] & {$[2.520]$} & [18.13] & [1.979] & [9.894] & [10.99] & [6.422] \\
\hline
\end{tabular}



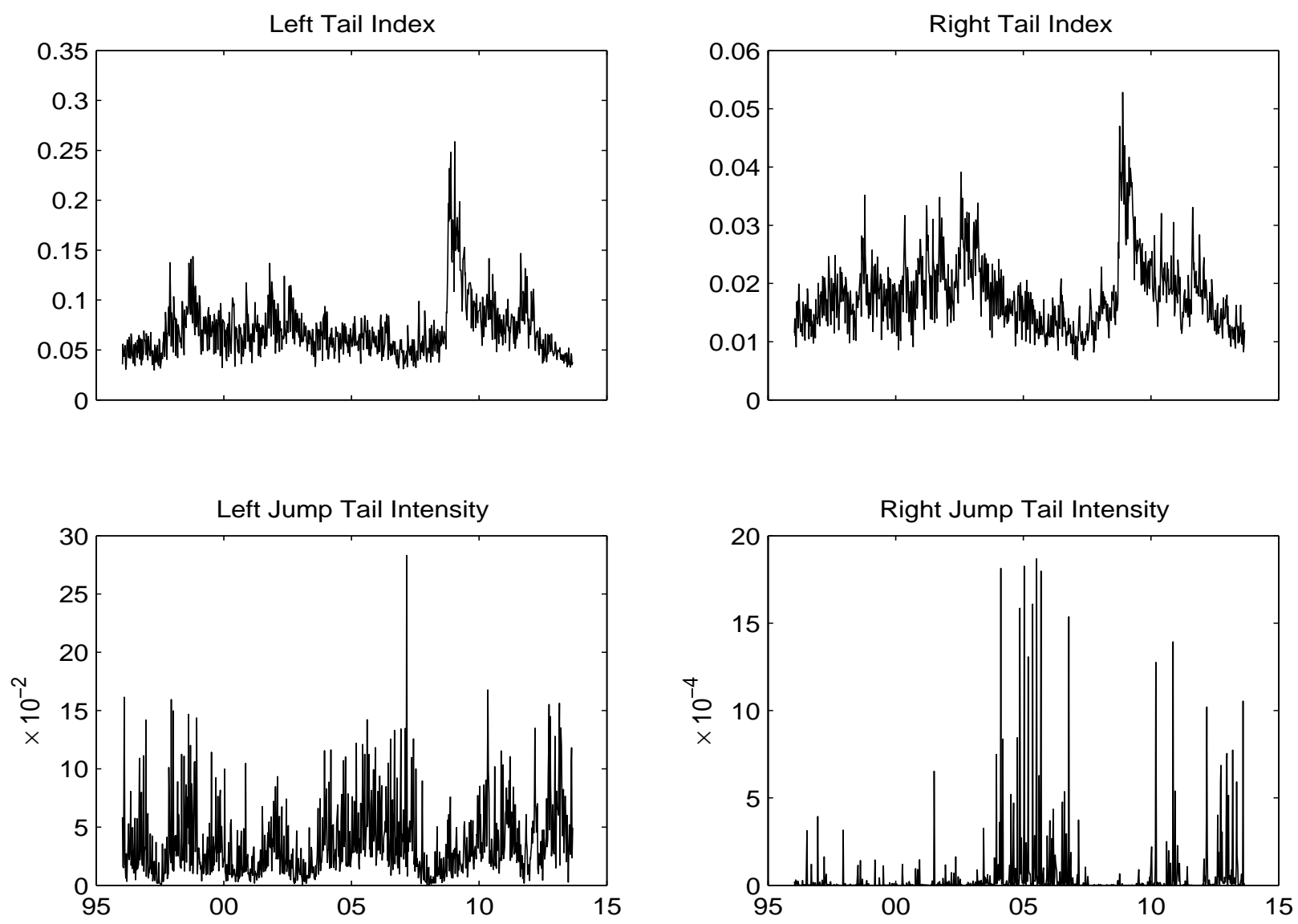

Figure 1: Tail Shape Parameters and Jump Intensities

The top two panels plot the estimated left and right tail indexes, $1 / \alpha_{t}^{ \pm}$, defined by the estimator in equation (3.3). The bottom two panels plot the estimated left and right jump intensities for "large" jumps beyond the threshold $k_{t}, L J I_{t}=\widehat{\phi}_{t}^{-} e^{-\widehat{\alpha}_{t}^{-}\left|k_{t}\right|} / \widehat{\alpha}_{t}^{-}$and $R J I_{t}=\widehat{\phi}_{t}^{+} e^{-\alpha_{t}^{+}\left|k_{t}\right|} / \widehat{\alpha}_{t}^{+}$, defined in equation (5.1). LJI and RJI are both reported in annualized square form. 

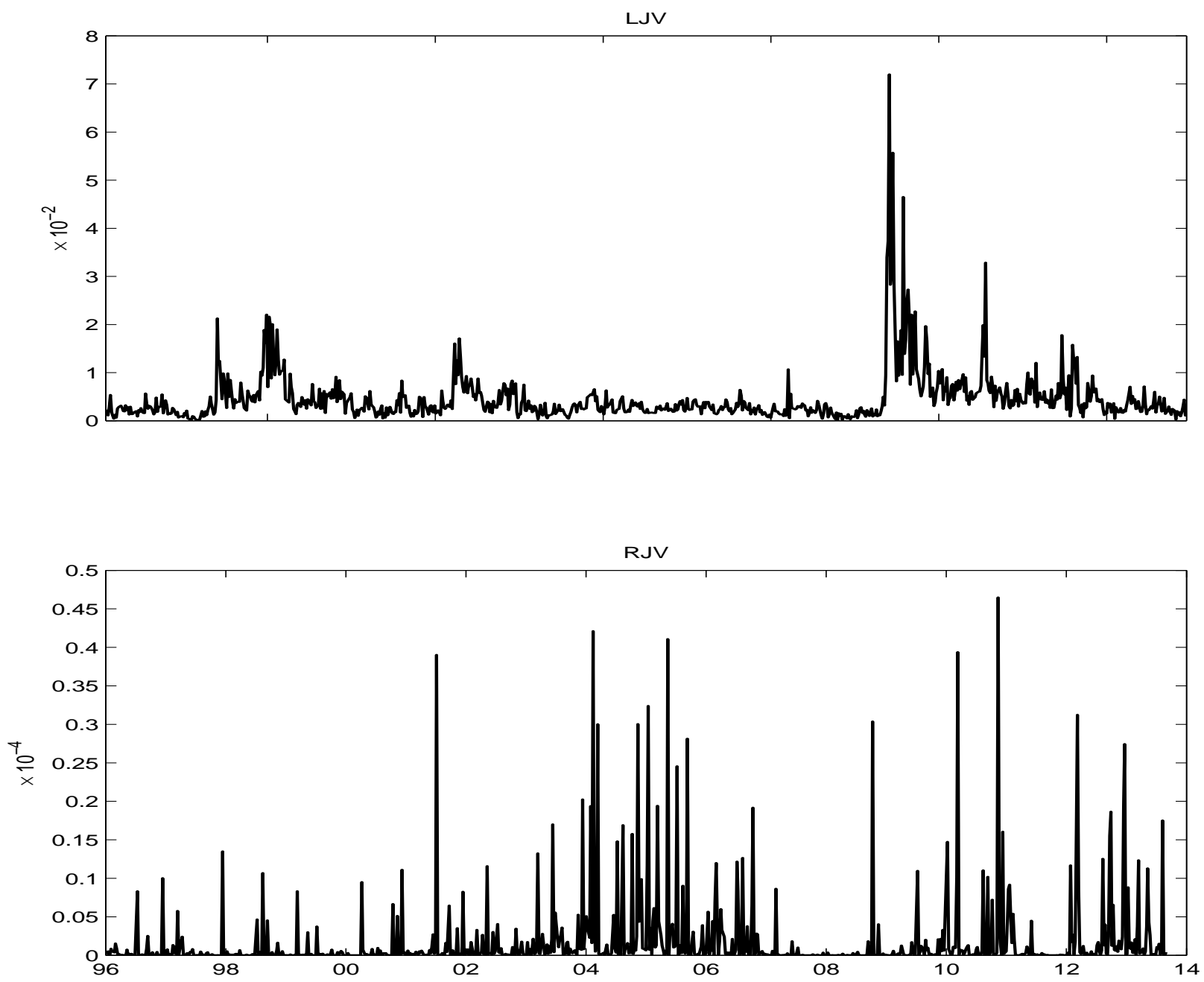

Figure 2: Jump Variation

This figure plots the left and right jump variation, $L J V_{t}$ and $R J V_{t}$, defined in equation (3.2). LJV and RJV are both reported in annualized square form. 

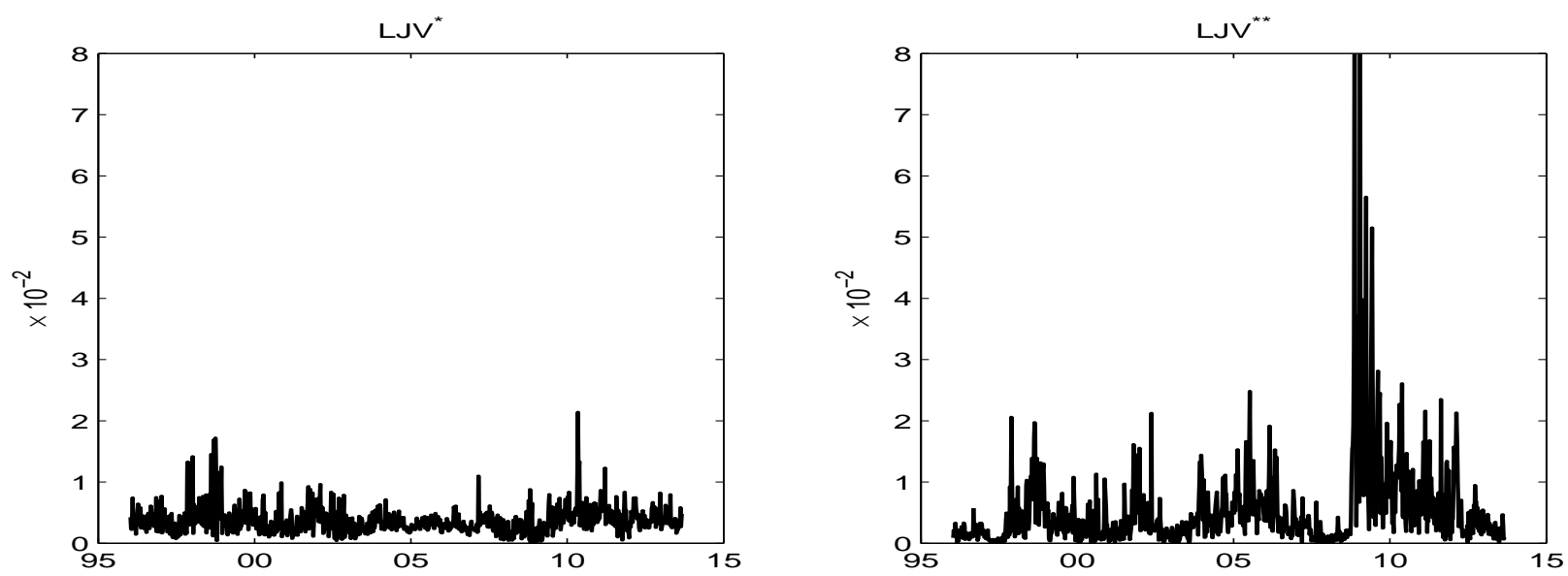

Figure 3: Left Jump Variation with Restricted Tail Parameters

The left panel plots the left jump variation $L J V_{t}^{*}$ obtained by restricting the "shape" parameter $\alpha^{-}$to be constant, but allowing $\phi_{t}^{-}$to change over time. The right panel shows $L J V_{t}^{* *}$ obtained by restricting the "level" parameter $\phi^{-}$to be constant, but allowing $\alpha_{t}^{-}$to be time-varying. The estimates are reported in annualized square form.
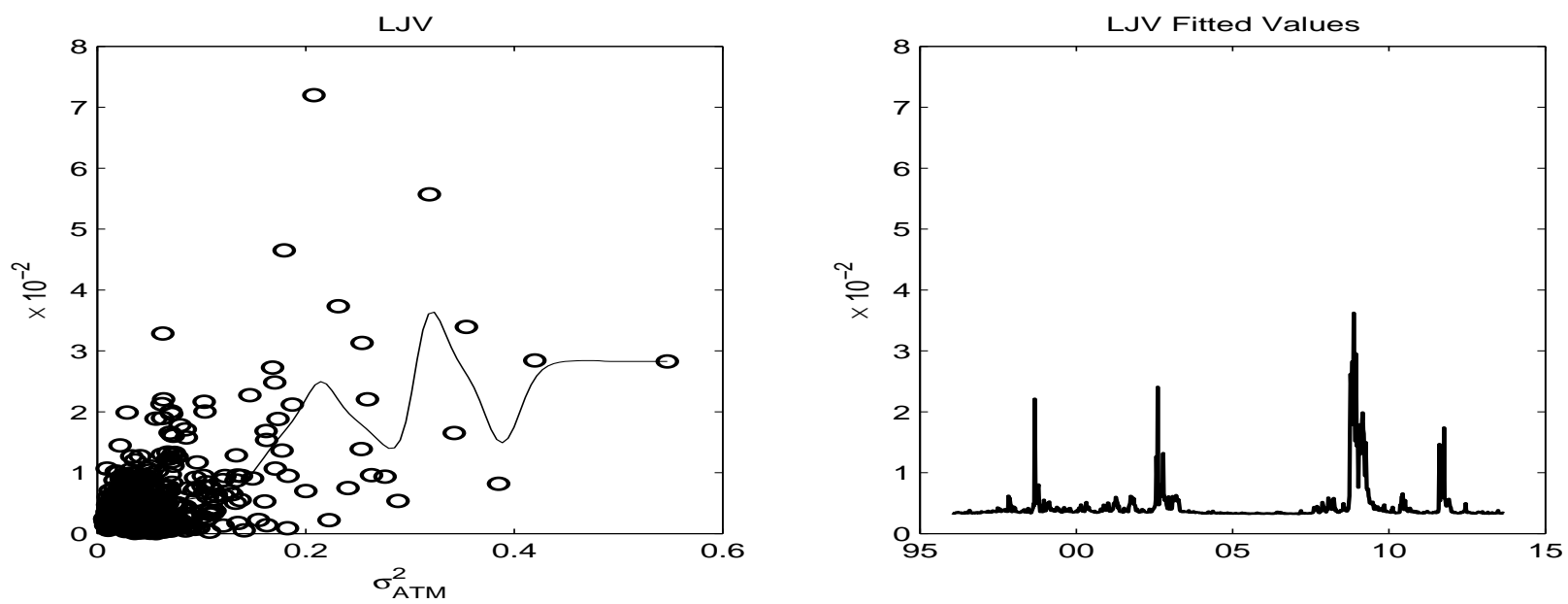

Figure 4: Nonparametric Regression of Left Jump Variation on Volatility

The left panel displays a scatterplot of $L J V_{t}$ against $\sigma_{A T M, t}^{2}$ (circles), along with the fit from a nonparametric kernel regression of $L J V_{t}$ on $\sigma_{A T M, t}^{2}$ (line), where $\sigma_{A T M, t}$ denotes the at-the-money implied volatility from the shortest-maturity options available on day $t$. The right panel shows a time series plot of the corresponding fitted values of $L J V_{t}$. 

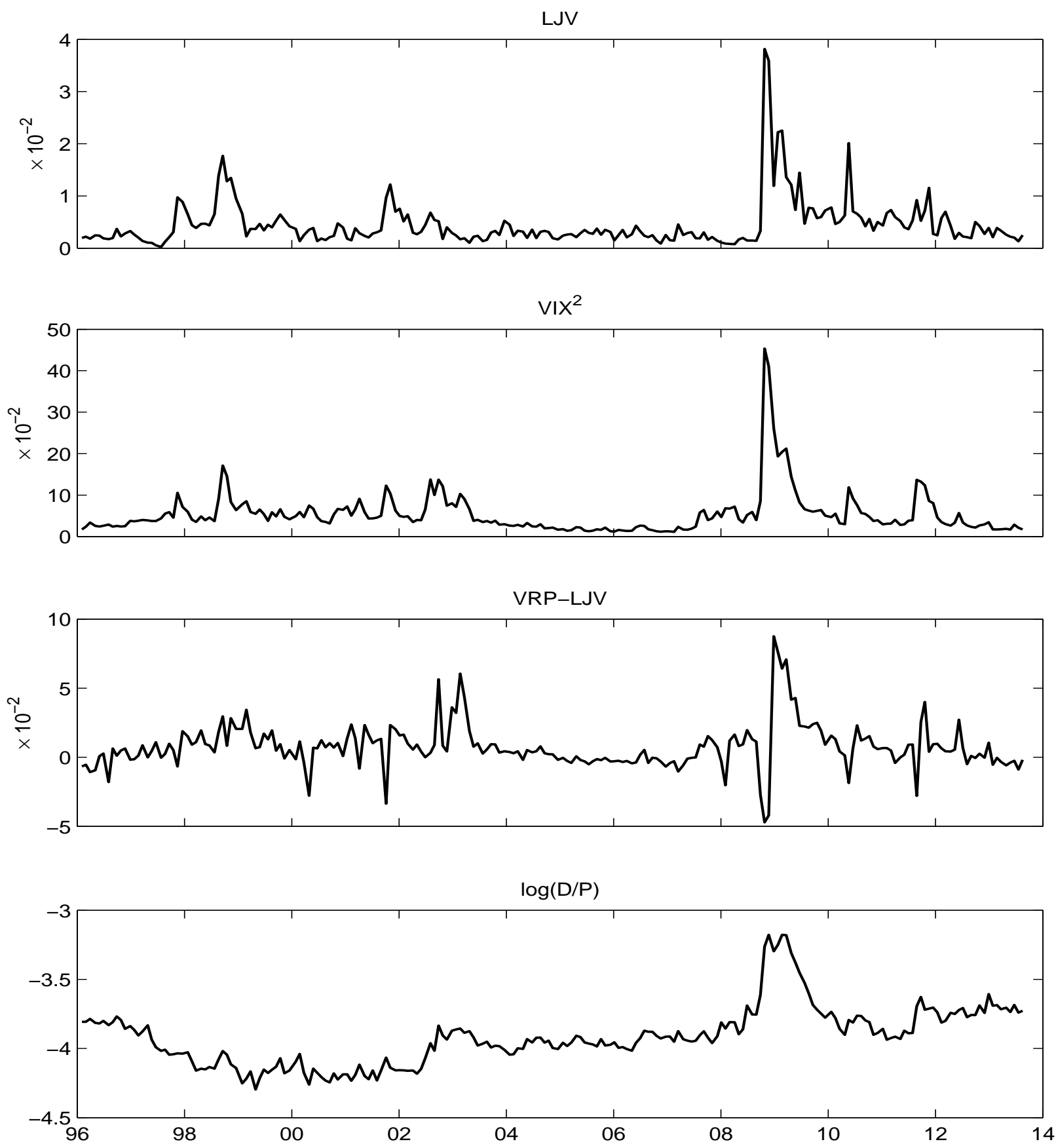

Figure 5: Predictor Variables

The series are observed monthly and span the period from January 1996 to August 2013. The top panel shows the estimated left jump tail variation measure $L J V_{t}$. The second panel shows the CBOE $V I X_{t}^{2}$ volatility index. The third panel plots the estimated variance risk premium $V R P_{t}$ minus $L J V_{t}$. All of the monthly variation measures are aggregated from the weekly option-based estimates and reported in annualized squared form. The bottom panel plots the log dividend-price ratio for the aggregate market $\log \left(D_{t} / P_{t}\right)$. 

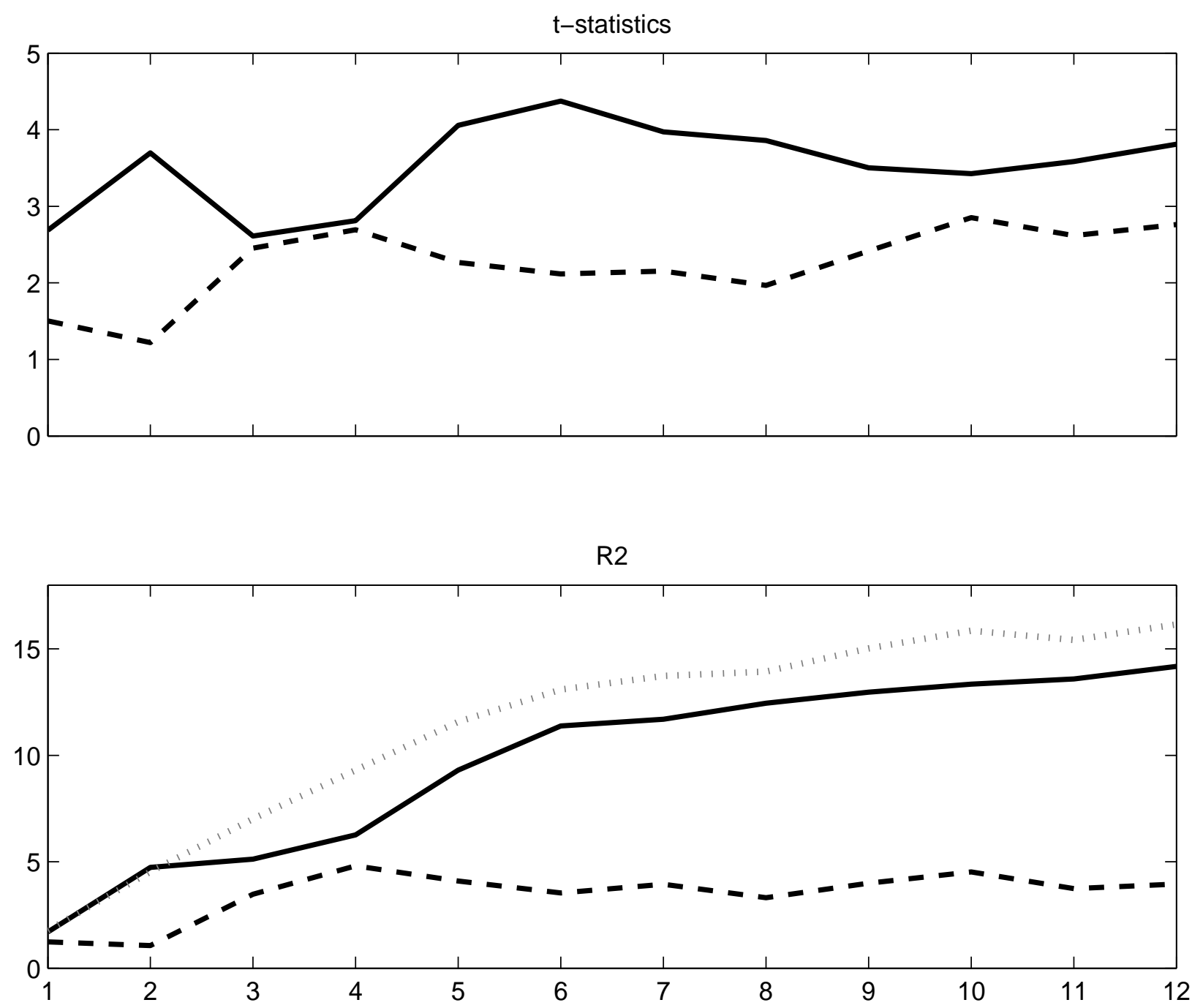

Figure 6: Market Return Predictability Regressions

The top panel shows the Newey-West t-statistics from from simple return predictability regressions for the aggregate market portfolio $M R K$ based on the left jump tail variation $L J V$ (solid line) and the difference between the variance risk premium and the left jump variation $V R P-L J V$ (dashed line). The bottom panel shows the corresponding $R^{2} \mathrm{~s}$, along with the $\mathrm{R}^{2} \mathrm{~s}$ from multiple regressions including both $L J V$ and $V R P-L J V$ (dotted line). 


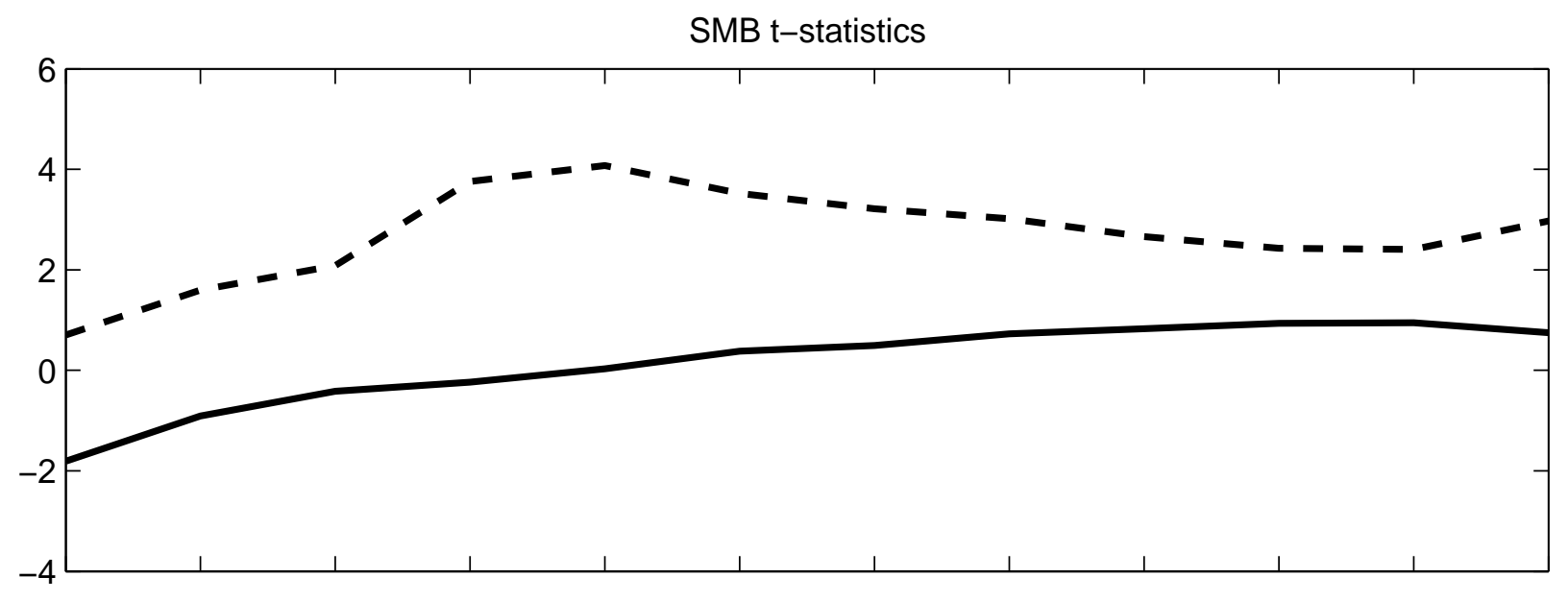

SMB R2

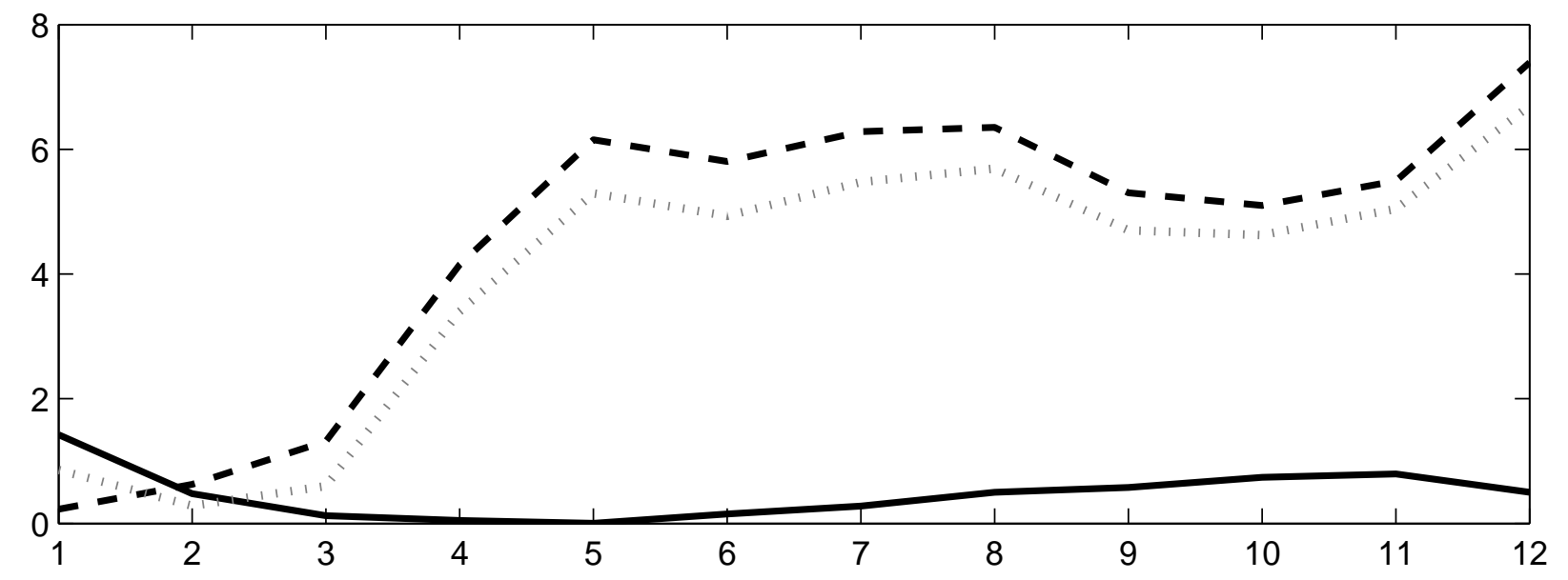

Figure 7: SMB Return Predictability Regressions

The top panel shows the Newey-West t-statistics from simple return predictability regressions for the Small Minus Big $(S M B)$ market capitalization sorted zero-cost portfolio based on the left jump tail variation $L J V$ (solid line) and the difference between the variance risk premium and the left jump variation $V R P-L J V$ (dashed line). The bottom panel shows the corresponding $R^{2} \mathrm{~s}$, along with the $\mathrm{R}^{2} \mathrm{~s}$ from multiple regressions including both $L J V$ and $V R P-L J V$ (dotted line). 
HML t-statistics

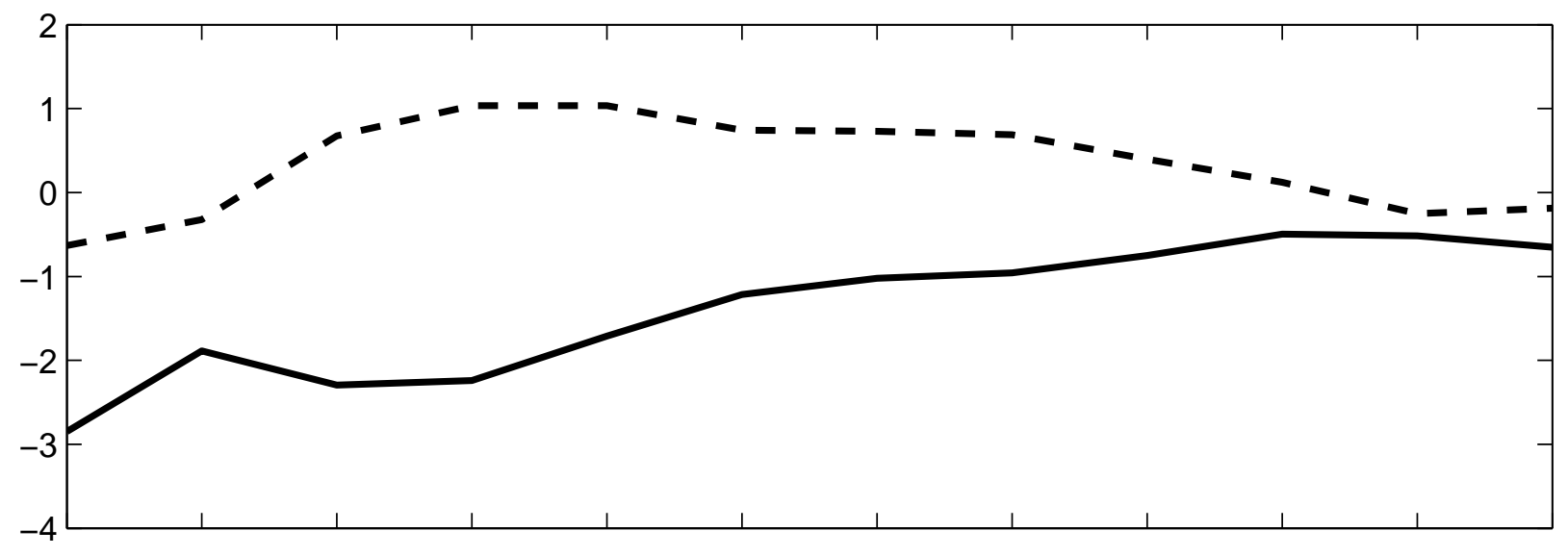

HML R2

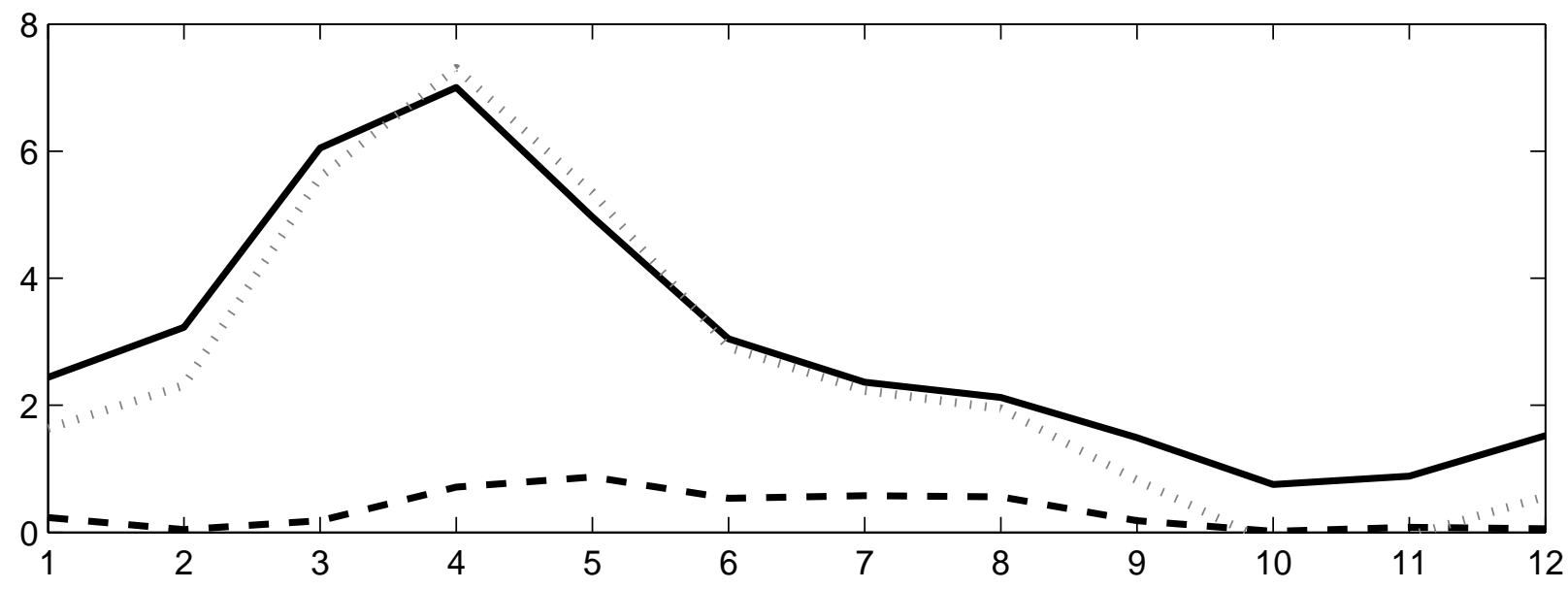

Figure 8: HML Return Predictability Regressions

The top panel shows the Newey-West t-statistics from simple return predictability regressions for the High Minus Low $(H M L)$ book-to-market sorted zero-cost portfolio based on the left jump tail variation $L J V$ (solid line) and the difference between the variance risk premium and the left jump variation $V R P-L J V$ (dashed line). The bottom panel shows the corresponding $R^{2} \mathrm{~s}$, along with the $\mathrm{R}^{2} \mathrm{~s}$ from multiple regressions including both $L J V$ and $V R P-L J V$ (dotted line). 
WML t-statistics

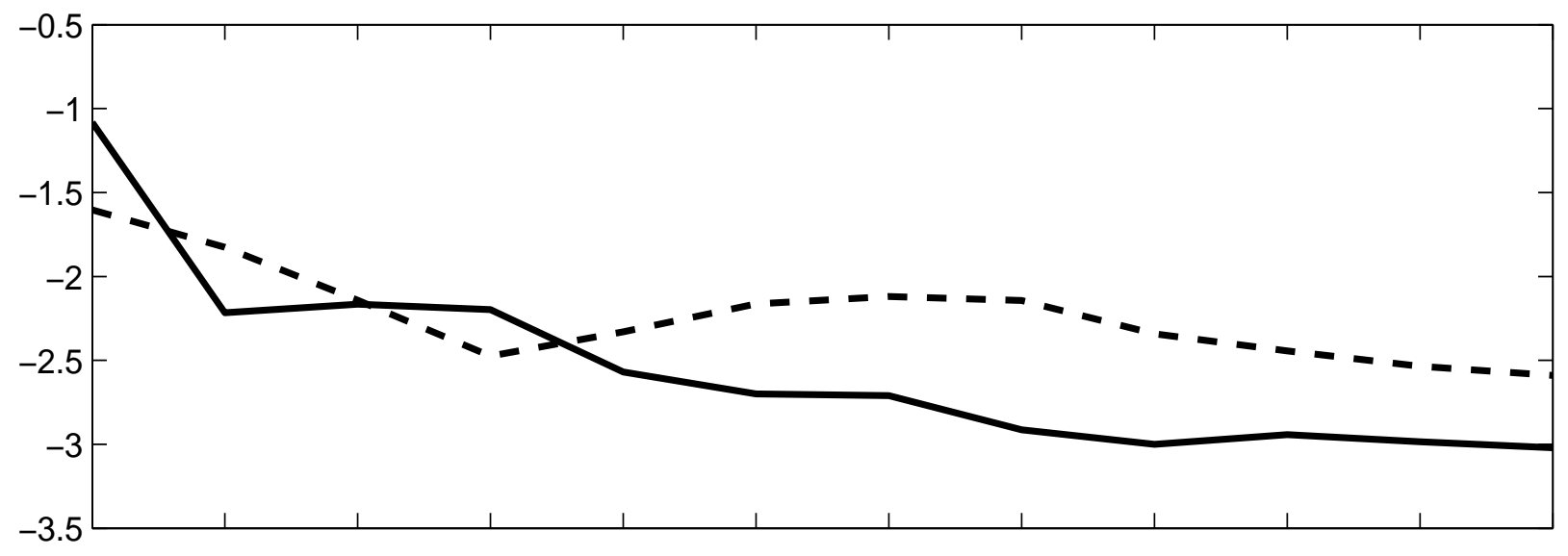

WML R2

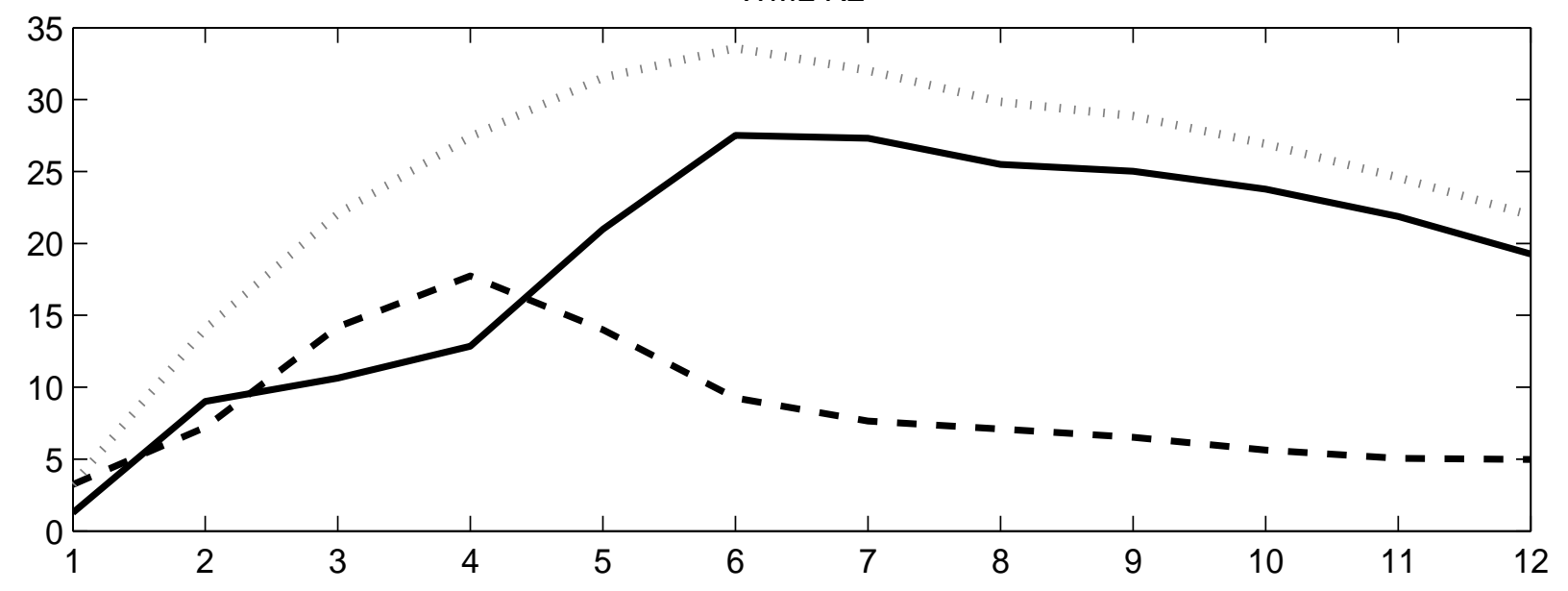

Figure 9: WML Return Predictability Regressions

The top panel shows the Newey-West t-statistics from simple return predictability regressions for the Winners Minus Losers $(W M L)$ sorted zero-cost portfolio based on the left jump tail variation $L J V$ (solid line) and the difference between the variance risk premium and the left jump variation $V R P-L J V$ (dashed line). The bottom panel shows the corresponding $R^{2} \mathrm{~s}$, along with the $\mathrm{R}^{2} \mathrm{~s}$ from multiple regressions including both $L J V$ and $V R P-L J V$ (dotted line). 
2014-32: $\quad$ Ulrich Hounyo: The wild tapered block bootstrap

2014-33: Massimiliano Caporin, Luca Corazzini and Michele Costola: Measuring the Behavioral Component of Financial Fluctuations: An Analysis Based on the S\&P 500

2014-34: $\quad$ Morten Ørregaard Nielsen: Asymptotics for the conditional-sum-of-squares estimator in multivariate fractional time series models

2014-35: Ulrich Hounyo: Bootstrapping integrated covariance matrix estimators in noisy jump-diffusion models with non-synchronous trading

2014-36: Mehmet Caner and Anders Bredahl Kock: Asymptotically Honest Confidence Regions for High Dimensional

2014-37: Gustavo Fruet Dias and George Kapetanios: Forecasting Medium and Large Datasets with Vector Autoregressive Moving Average (VARMA) Models

2014-38: Søren Johansen: Times Series: Cointegration

2014-39: Søren Johansen and Bent Nielsen: Outlier detection algorithms for least squares time series regression

2014-40: $\quad$ Søren Johansen and Lukasz Gatarek: Optimal hedging with the cointegrated vector autoregressive model

2014-41: $\quad$ Laurent Callot and Johannes Tang Kristensen: Vector Autoregressions with Parsimoniously Time Varying Parameters and an Application to Monetary Policy

2014-42: $\quad$ Laurent A. F. Callot, Anders B. Kock and Marcelo C. Medeiros: Estimation and Forecasting of Large Realized Covariance Matrices and Portfolio Choice

2014-43: $\quad$ Paolo Santucci de Magistris and Federico Carlini: On the identification of fractionally cointegrated VAR models with the $F(d)$ condition

2014-44: $\quad$ Laurent Callot, Niels Haldrup and Malene Kallestrup Lamb: Deterministic and stochastic trends in the Lee-Carter mortality model

2014-45: $\quad$ Nektarios Aslanidis, Charlotte Christiansen, Neophytos Lambertides and Christos S. Savva: Idiosyncratic Volatility Puzzle: Influuence of Macro-Finance Factors

2014-46: Alessandro Giovannelli and Tommaso Proietti: On the Selection of Common Factors for Macroeconomic Forecasting

2014-47: $\quad$ Martin M. Andreasen and Andrew Meldrum: Dynamic term structure models: The best way to enforce the zero lower bound

2014-48: $\quad$ Tim Bollerslev, Sophia Zhengzi Li and Viktor Todorov: Roughing up Beta: Continuous vs. Discontinuous Betas, and the Cross-Section of Expected Stock Returns

2914-49: $\quad$ Tim Bollerslev, Viktor Todorov and Lai Xu: Tail Risk Premia and Return Predictability 